\title{
Eating from the wild: diversity of wild edible plants used by Tibetans in Shangri-la region, Yunnan, China
}

\author{
Yan Ju', Jingxian Zhuo ${ }^{2}$, Bo Liu' ${ }^{1}$ and Chunlin Long ${ }^{1,3^{*}}$
}

\begin{abstract}
Background: Locally harvested wild edible plants (WEPs) provide food as well as cash income for indigenous people and are of great importance in ensuring global food security. Some also play a significant role in maintaining the productivity and stability of traditional agro-ecosystems. Shangri-la region of Yunnan Province, SW China, is regarded as a biodiversity hotspot. People living there have accumulated traditional knowledge about plants. However, with economic development, WEPs are threatened and the associated traditional knowledge is in danger of being lost. Therefore, ethnobotanical surveys were conducted throughout this area to investigate and document the wild edible plants traditionally used by local Tibetan people.
\end{abstract}

Methods: Twenty-nine villages were selected to carry out the field investigations. Information was collected using direct observation, semi-structured interviews, individual discussions, key informant interviews, focus group discussions, questionnaires and participatory rural appraisal (PRA).

Results: Information about 168 wild edible plant species in 116 genera of 62 families was recorded and specimens were collected. Most species were edible greens (80 species) or fruits (78). These WEPs are sources for local people, especially those living in remote rural areas, to obtain mineral elements and vitamins. More than half of the species (70\%) have multiple use(s) besides food value. Some are crop wild relatives that could be used for crop improvement. Several also have potential values for further commercial exploitation. However, the utilization of WEPs and related knowledge are eroding rapidly, especially in the areas with convenient transportation and booming tourism.

Conclusion: Wild food plants species are abundant and diverse in Shangri-la region. They provide food and nutrients to local people and could also be a source of cash income. However, both WEPs and their associated indigenous knowledge are facing various threats. Thus, conservation and sustainable utilization of these plants in this area are of the utmost importance. Documentation of these species may provide basic information for conservation, possibly further exploitation, and will preserve local traditional knowledge.

Keywords: Wild edible plants, Traditional knowledge, Biodiversity, Ethnobotany, Shangri-la region

\footnotetext{
* Correspondence: chunlinlong@hotmail.com

${ }^{1}$ College of Life and Environmental Sciences, Minzu University of China,

Beijing 100081, PR China

${ }^{3}$ Kunming Institute of Botany, Chinese Academy of Sciences, Kunming

650201, PR China

Full list of author information is available at the end of the article
} 


\section{Background}

Wild edible plants (WEPs) refer to species that are harvested or collected from their wild natural habitats and used as food for human consumption [1-3]. They provide staple food for indigenous people, serve as supplementary food for non-indigenous people and are one of the primary sources of cash income for poor communities [4-6]. WEPs play an important role in ensuring food security and improve the nutrition in the diets of many people in developing countries $[1,5]$. They are potential sources of species for domestication and provide valuable genetic traits for developing new crops through breeding and selection $[7,8]$.

Although domesticated plants are the main source of food and income for people in rural areas, they are not able to meet the annual food requirements [9-11]. Thus, the collection and consumption of wild edible plants has been "a way of life" to supplement dietary requirements for many rural populations throughout the world $[5,12]$. However, due to social change and acculturation processes, indigenous knowledge (or traditional knowledge) about the use of wild edible species is declining and even vanishing with modernization and increasing contacts with western lifestyles [13]. Meanwhile, the loss of traditional knowledge has also been recognized as one of the major factors that have negative effects on the conservation of biological diversity [14]. Thus, it is becoming urgent to document and revitalize traditional knowledge of WEPs to preserve genetic and cultural diversity $[12,15,16]$. China is renowned for its wide use of wild harvested resources in the human diet, and many studies have focused on wild edible plants [17-28]. These ethnobotanical surveys not only play an important role in conserving traditional knowledge associated with WEPs, but also contribute to nutritional analysis of the most widely used species $[1,13]$. Nutritional analyses may provide significant information for the utilization of those species that have the best nutritional values, thus helping to maintain dietary diversity and improve local food security $[1,2,15]$.

Diqing Tibetan Autonomous Prefecture of Yunnan Province, commonly known as the Shangri-la region, belongs to the world-famous area called Three Parallel Rivers (Nujiang River, Lancang River and Jinsha River). It is the core of the eastern Himalayas and is regarded as a biodiversity hotspot [29]. Because of its complex topography and high diversity of climates, abundant plant and animal species are distributed in this area [30,31]. Although Tibetans account for about $32.36 \%$ of the total population of the whole prefecture and have a relatively well-preserved and distinct cultural identity, there are also Lisu, Han, Naxi, Yi as well as Bai populations, among whom mutual cultural influences have existed for a long time [30,31]. Furthermore, the diet of local
Tibetan people differs somewhat from that of Tibetans in Xizang Autonomous Region. People living in the Tibetan Plateau have a limited range of food choices. The staple traditional diet includes Tsampa (made from hull-less barley), yak meat, mutton, buttered tea, sweet tea, barley wine and yogurt [32]. They seldom eat vegetables or fruits. On the other hand, because plant resources in Diqing Prefecture are more plentiful, and local Tibetans are influenced by other nationalities, they not only cultivate various crops, but also collect wild edible plants as supplementary food. These WEPs provide various microelements, and are also an important feature of local agrobiodiversity in which Tibetans have traditionally lived. However, the ecology of Diqing Tibetan Autonomous Prefecture is very fragile, and agrobiodiversity is being rapidly lost due to many natural and human caused factors [33-35]. Many precious plant resources that may have potential for future sustainable development are vanishing before they have been discovered. The reduction of plant diversity also leads to the extinction of the associated indigenous knowledge [36]. Thus, documentation and evaluation of edible plants and relevant local knowledge is urgently needed. This work may guide proper conservation and sustainable utilization of those wild food plants and related indigenous knowledge.

Although there are several ethnobotanical studies concerning wild food plants used by ethnic minorities, such as Mongolians [18,19], Miao in Hunan Province [21] and various ethnic groups in Yunnan Province [5,17,26-28], to our knowledge, information on WEPs of the Shangri-la region used by Tibetans has not previously been documented. In order to fill this gap, ethnobotanical surveys were conducted throughout the prefecture. Scientific and local names, plant parts used, modes of preparation, seasonality patterns in collection and use, and commercialization possibilities of the WEPs are presented in this paper.

\section{Methods}

\section{Study area}

The study was carried out in Diqing Tibetan Autonomous Prefecture, northwest Yunnan, situated in the south of the Qinghai-Tibet Plateau of the eastern Himalayas, at the junction of Yunnan, Tibet and Sichuan Provinces (between $98^{\circ} 35^{\prime}-100^{\circ} 19^{\prime} \mathrm{E}$ and $26^{\circ} 52^{\prime}-29^{\circ} 16^{\prime} \mathrm{N}$ ) (Figure 1). Three counties, Shangri-la, Deqin and Weixi are administered by the prefecture, with a total area of 23,870 square kilometers and a population of about 400,000. The terrain is higher in the north and lower in the south. The lowest altitude, $1,480 \mathrm{~m}$ is at the junction of the Biyu and Lancang Rivers in Weixi County, and the highest altitude, 6,740 $\mathrm{m}$ is Kawagebo Peak of the Meili Snow Mountains. The climate of Diqing is divided into five zones: 1) 


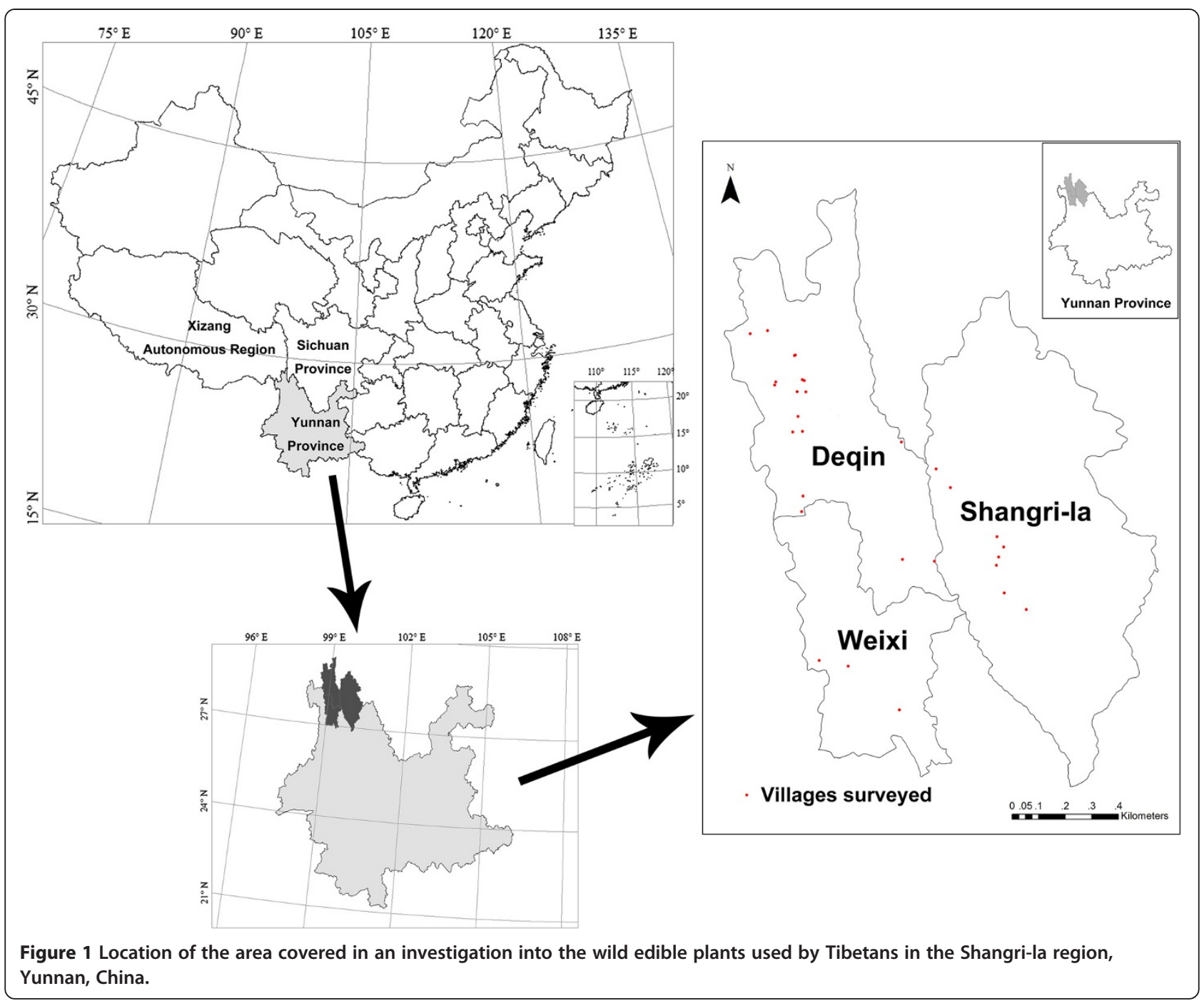

northern subtropical and warm temperate (below $2500 \mathrm{~m})$; 2) temperate $(2500-3000 \mathrm{~m})$; 3) cold temperate (3000-4000 m); 4) frigid (4000-5000 m); and 5) glacier (above $5000 \mathrm{~m}$ ). Abundant plant resources are distributed in this area because of its unique geographical location and climate diversity [31].

\section{Field survey and data collection}

Prior to our field work, relevant literature was consulted to obtain information on the topography, climate, and local culture of Diqing Prefecture, this was helpful in choosing the specific study sites [31]. Field studies were carried out during three visits in March, July and August, 2012. After considering the terrain and climate condition, 29 villages belonging to three counties (8 in Shangri-la, 3 in Weixi and 18 in Deqin) and located in high mountains as well as lower river valleys were randomly selected to carry out ethnobotanical investigation
(Table 1). Two-hundred and eighty-two randomly selected households (eight to ten people per village) were surveyed. Ethnobotanical data were collected through different interview methods (participatory rural appraisal (PRA), direct observation, semi-structured interviews, key informant interviews, individual discussions, focus group discussions and questionnaires) [37-40].

During our survey, the local Tibetan pronunciations, parts used, collection period and preparation methods plants were recorded. Because local Tibetan pronunciations differ from the formal Tibetan pronunciation of Xizang Autonomous Region, and the names of some species were even pronounced the same as in Mandarin Chinese, we recorded the names phonetically exactly as they were spoken to us. Most Tibetans in Diqing Prefecture, especially the official workers, students and traders can speak basic Mandarin, therefore our interviews were in Mandarin and did not use interpreters. 
Table 1 Villages surveyed in investigations of wild edible plants used by Tibetans in Shangri-la region, Yunnan Province, China

\begin{tabular}{|c|c|c|c|c|}
\hline No. & Name of village & Latitude (north) & Longitude (east) & Altitude(m) \\
\hline 1 & Laza Village, Shangri-la County & $27^{\circ} 45^{\prime} 39.6^{\prime \prime}$ & $99^{\circ} 40^{\prime} 22.8^{\prime \prime}$ & 3320 \\
\hline 2 & Jiefang Village, Shangri-la County & $27^{\circ} 51^{\prime} 46.8^{\prime \prime}$ & $99^{\circ} 41^{\prime} 56.4^{\prime \prime}$ & 3280 \\
\hline 3 & Nishi Village, Shangri-la County & $27^{\circ} 47^{\prime} 21.8^{\prime \prime}$ & $99^{\circ} 40^{\prime} 59.5^{\prime \prime}$ & 3290 \\
\hline 4 & Kaisong Village, Shangri-la County & $27^{\circ} 53^{\prime} 49.6^{\prime \prime}$ & $99^{\circ} 38^{\prime} 21.5^{\prime \prime}$ & 3270 \\
\hline 5 & Dala Village, Shangri-la County & $27^{\circ} 31^{\prime} 12^{\prime \prime}$ & $99^{\circ} 57^{\prime} 36^{\prime \prime}$ & 3370 \\
\hline 6 & Xiaozhongdian Village, Shangri-la County & $27^{\circ} 34^{\prime} 12^{\prime \prime}$ & $99^{\circ} 47^{\prime} 59^{\prime \prime}$ & 3260 \\
\hline 7 & Xingfu Village, Shangri-la County & $28^{\circ} 8^{\prime} 27.6^{\prime \prime}$ & $99^{\circ} 25^{\prime} 58.8^{\prime \prime}$ & 2230 \\
\hline 8 & Nixi Village, Shangri-la County & $28^{\circ} 4^{\prime} 1.2^{\prime \prime}$ & $99^{\circ} 29^{\prime} 34.8^{\prime \prime}$ & 3170 \\
\hline 9 & Laohao Village, Weixi County & $27^{\circ} 10^{\prime} 54.1^{\prime \prime}$ & $99^{\circ} 17^{\prime} 20.4^{\prime \prime}$ & 2260 \\
\hline 10 & Gongyuan Village, Weixi County & $27^{\circ} 21^{\prime} 50.35^{\prime \prime}$ & $99^{\circ} 5^{\prime} 11.45^{\prime \prime}$ & 1690 \\
\hline 11 & Biluo Village, Weixi County & $27^{\circ} 25^{\prime} 22.8^{\prime \prime}$ & $99^{\circ} 1{ }^{\prime} 58.8^{\prime \prime}$ & 2630 \\
\hline 12 & Feilaisi Village, Deqin County & $28^{\circ} 26^{\prime} 31.2^{\prime \prime}$ & $98^{\circ} 52^{\prime} 44.4^{\prime \prime}$ & 3390 \\
\hline 13 & Wunongding Village, Deqin County & $28^{\circ} 26^{\prime} 56.4^{\prime \prime}$ & $98^{\circ} 54^{\prime} 46.8^{\prime}$ & 3530 \\
\hline 14 & Mingyong Village, Deqin County & $28^{\circ} 28^{\prime} 84^{\prime \prime}$ & $98^{\circ} 47^{\prime} 42^{\prime \prime}$ & 2270 \\
\hline 15 & Adunzi Village, Deqin County & $28^{\circ} 29^{\prime} 13.6^{\prime \prime}$ & $98^{\circ} 54^{\prime} 38.9^{\prime \prime}$ & 3290 \\
\hline 16 & Gusong Village, Deqin County & $28^{\circ} 29^{\prime} 37.7^{\prime \prime}$ & $98^{\circ} 54^{\prime} 10.1^{\prime \prime}$ & 3590 \\
\hline 17 & Adong Village, Deqin County & $28^{\circ} 45^{\prime} 46.8^{\prime \prime}$ & $98^{\circ} 39^{\prime} 14.4^{\prime \prime}$ & 2690 \\
\hline 18 & Hongpo Village, Deqin County & $28^{\circ} 17^{\prime} 2.4^{\prime \prime}$ & $98^{\circ} 54^{\prime} 18^{\prime \prime}$ & 2810 \\
\hline 19 & Guonian Village, Deqin County & $28^{\circ} 17^{\prime} 16.71^{\prime \prime}$ & $98^{\circ} 51^{\prime} 49.61^{\prime \prime}$ & 2130 \\
\hline 20 & Jiulongding Village, Deqin County & $28^{\circ} 20^{\prime} 42^{\prime \prime}$ & $98^{\circ} 53^{\prime} 13.2^{\prime \prime}$ & 2570 \\
\hline 21 & Sinong Village, Deqin County & $28^{\circ} 29^{\prime} 9.18^{\prime}$ & $98^{\circ} 47^{\prime} 33.42^{\prime}$ & 2320 \\
\hline 22 & Badong Village, Deqin County & $27^{\circ} 57^{\prime} 39.6^{\prime \prime}$ & $98^{\circ} 54^{\prime} 0^{\prime \prime}$ & 2240 \\
\hline 23 & Cizhong Village, Deqin County & $28^{\circ} 01^{\prime} 16.44^{\prime}$ & $98^{\circ} 54^{\prime} 16.14^{\prime}$ & 1970 \\
\hline 24 & Gongka Village, Deqin County & $28^{\circ} 35^{\prime} 27.6^{\prime \prime}$ & $98^{\circ} 52^{\prime} 12^{\prime \prime}$ & 3080 \\
\hline 25 & Jiunong Village, Deqin County & $28^{\circ} 43^{\prime} 28.77^{\prime \prime}$ & $98^{\circ} 41^{\prime} 4.76^{\prime \prime}$ & 3160 \\
\hline 26 & Luwa Village, Deqin County & $28^{\circ} 40^{\prime} 30^{\prime \prime}$ & $98^{\circ} 41^{\prime} 38.4^{\prime \prime}$ & 2290 \\
\hline 27 & Xiaruo Village, Deqin County & $27^{\circ} 48^{\prime} 3.77^{\prime \prime}$ & $99^{\circ} 18^{\prime} 5.11^{\prime \prime}$ & 2040 \\
\hline 28 & Tuoding Village, Deqin County & $27^{\circ} 46^{\prime} 10.9^{\prime}$ & $99^{\circ} 25^{\prime} 37.2^{\prime}$ & 1940 \\
\hline 29 & Benzilan Village, Deqin County & $28^{\circ} 14^{\prime} 36.83^{\prime \prime}$ & $99^{\circ} 18^{\prime} 7.43^{\prime \prime}$ & 2150 \\
\hline
\end{tabular}

Specimens were examined and identified by the authors and other taxonomists and will be deposited in the Herbarium of the Minzu University of China (Beijing).

\section{Results and discussion}

\section{Wild food plant diversity and frequently utilized species}

The study area is floristically rich and has a large number of useful WEP species. The 168 species documented include angiosperms (153 spp.), gymnosperms (4), pteridophytes (4), algae (2) and lichens (5) (Table 2), of which 41.1\% are endemic to China and $11.9 \%$ endemic to northwestern Yunnan Province. Details of utilization are given in Table 3 (plants mentioned only by one informant are not documented in this list). The average number of species mentioned per informant is around ca. 8 species. Plants belonging to 62 families and 116 genera are

Table 2 Taxonomic distribution of wild edible plants used by Tibetans in Shangri-la region, Yunnan Province, China

\begin{tabular}{llll}
\hline Plant group & Number of species & Number of genera & Number of families \\
\hline Angiosperm & 153 & 101 & 47 \\
\hline Gymnosperm & 4 & 4 & 4 \\
\hline Pteridophyte & 4 & 4 & 4 \\
\hline Algae & 2 & 2 & 2 \\
\hline Lichen & 5 & 5 & 5 \\
\hline Total & 168 & 116 & 62 \\
\hline
\end{tabular}


Table 3 Wild edible plants used by the Tibetans in Shangri-la region, Yunnan Province, China

\begin{tabular}{|c|c|c|c|c|c|c|c|c|}
\hline Latin name & Local name & Family name & Distribution & Parts used & Local use (edible only) & $\begin{array}{l}\text { Collection } \\
\text { period }\end{array}$ & Additional local use(s) & Frequency \\
\hline $\begin{array}{l}\text { Actinidia arguta } \\
\text { (Siebold et Zucc.) } \\
\text { Planch. ex Miq. }\end{array}$ & Zhemenkoubu & Actinidiaceae & $\begin{array}{l}\text { Shangri-la, } \\
\text { Weixi and } \\
\text { Deqin }\end{array}$ & Fruits & ripe fruits eaten fresh. & Aug-Sept & $\begin{array}{l}\text { Whole plants used as hedge } \\
\text { plants. }\end{array}$ & *** \\
\hline $\begin{array}{l}\text { Actinidia pilosula } \\
\text { (Finet et } \\
\text { Gagnep.) Stapf } \\
\text { ex Hand.-Mazz. }\end{array}$ & Zhemenkoubu & Actinidiaceae & $\begin{array}{l}\text { Shangri-la, } \\
\text { Weixi and } \\
\text { Deqin }\end{array}$ & Fruits & ripe fruits eaten fresh. & Aug-Sept & $\begin{array}{l}\text { Whole plants used as hedge } \\
\text { plants. }\end{array}$ & $* *$ \\
\hline $\begin{array}{l}\text { Actinidia venosa } \\
\text { Rehder }\end{array}$ & Zhemenkoubu & Actinidiaceae & $\begin{array}{l}\text { Shangri-la, } \\
\text { Weixi and } \\
\text { Deqin }\end{array}$ & Fruits & ripe fruits eaten fresh. & Aug-Sept & $\begin{array}{l}\text { Whole plants used as hedge } \\
\text { plants. }\end{array}$ & $* *$ \\
\hline $\begin{array}{c}\text { Adenophora } \\
\text { khasiana (Hook. f. } \\
\text { et Thomson) } \\
\text { Collett et Hemsl. }\end{array}$ & Zheibamiedu & Campanulaceae & $\begin{array}{l}\text { Weixi and } \\
\text { Deqin }\end{array}$ & Roots & stewed with meat and eaten as tonic. & Jul-Sept & $\begin{array}{l}\text { Flowers and stems used for } \\
\text { weisang. Aerial parts used as } \\
\text { fodder. Roots used to treat } \\
\text { cough and clearing heat. }\end{array}$ & $* * *$ \\
\hline $\begin{array}{l}\text { Alectoria sulcata } \\
\text { Nyl. }\end{array}$ & Shuhua & Usneaceae & $\begin{array}{l}\text { Shangri-la, } \\
\text { Weixi and } \\
\text { Deqin }\end{array}$ & Whole plant & stir-fried & Jul-Sep & & * \\
\hline $\begin{array}{l}\text { Allium hookeri } \\
\text { Thwaites var. } \\
\text { muliense Airy- } \\
\text { Shaw }\end{array}$ & Rijicai & Liliaceae & $\begin{array}{l}\text { Shangri-la, } \\
\text { Weixi and } \\
\text { Deqin }\end{array}$ & Aerial parts & stir-fried or added to soups & May-Aug & & $* * *$ \\
\hline $\begin{array}{l}\text { Allium ovalifolium } \\
\text { Hand.-Mazz. }\end{array}$ & Rijicai & Liliaceae & $\begin{array}{l}\text { Shangri-la, } \\
\text { Weixi and } \\
\text { Deqin }\end{array}$ & Aerial parts & stir-fried or added to soups & May-Aug & & $* * * * *$ \\
\hline $\begin{array}{l}\text { Allium trifurcatum } \\
\text { (F. T. Wang et T. } \\
\text { Tang) J. M. Xu }\end{array}$ & Rijicai & Liliaceae & $\begin{array}{l}\text { Shangri-la, } \\
\text { Weixi and } \\
\text { Deqin }\end{array}$ & Aerial parts & stir-fried or added to soups & May-Aug & & **** \\
\hline $\begin{array}{l}\text { Amaranthus } \\
\text { caudatus L. }\end{array}$ & Yani & Amaranthaceae & $\begin{array}{l}\text { Shangri-la, } \\
\text { Weixi and } \\
\text { Deqin }\end{array}$ & $\begin{array}{l}\text { Young } \\
\text { stems and } \\
\text { leaves }\end{array}$ & stir-fried or added to soups & Jun-Jul & Aerial parts used as fodder. & $* * *$ \\
\hline $\begin{array}{c}\text { Amaranthus } \\
\text { hypochondriacus } \\
\text { L. }\end{array}$ & Yani & Amaranthaceae & $\begin{array}{l}\text { Shangri-la, } \\
\text { Weixi and } \\
\text { Deqin }\end{array}$ & $\begin{array}{l}\text { Young } \\
\text { stems and } \\
\text { leaves }\end{array}$ & stir-fried or added to soups & Jun-Jul & Aerial parts used as fodder. & $* * *$ \\
\hline $\begin{array}{l}\text { Amygdalus mira } \\
\text { (Koehne) Ricker }\end{array}$ & $\begin{array}{l}\text { Yemaotao; } \\
\text { Kamu }\end{array}$ & Rosaceae & $\begin{array}{l}\text { Shangri-la, } \\
\text { Weixi and } \\
\text { Deqin }\end{array}$ & Fruits & eaten fresh. & Jul-Aug & $\begin{array}{l}\text { Seeds used to relieve a cough } \\
\text { and cure injuries. }\end{array}$ & $* * *$ \\
\hline $\begin{array}{l}\text { Anemone rivularis } \\
\text { Buch.-Ham. ex } \\
\text { DC. }\end{array}$ & Huzhangcao & Ranunculaceae & Weixi & Roots & stewed with meat and eaten as tonic & Jun-Sept & $\begin{array}{l}\text { Roots used to treat bronchitis. } \\
\text { Whole plant used as } \\
\text { ornamental. }\end{array}$ & * \\
\hline $\begin{array}{l}\text { Aralia caesia } \\
\text { Hand.-Mazz. }\end{array}$ & Shutoucai & Araliaceae & Shangri-la & $\begin{array}{l}\text { Young } \\
\text { leaves and } \\
\text { leaf buds }\end{array}$ & stir-fried or eaten fresh & Apr-May & & $* * * *$ \\
\hline
\end{tabular}


Table 3 Wild edible plants used by the Tibetans in Shangri-la region, Yunnan Province, China (Continued)

\begin{tabular}{|c|c|c|c|c|c|c|c|c|}
\hline Aralia chinensis $\mathrm{L}$. & Gege & Araliaceae & $\begin{array}{l}\text { Shangri-la, } \\
\text { Weixi and } \\
\text { Deqin }\end{array}$ & $\begin{array}{l}\text { Young } \\
\text { leaves and } \\
\text { leaf buds }\end{array}$ & stir-fried or eaten fresh & Apr-May & Bark used for weisang & $* * * * *$ \\
\hline Arctium lappa L. & Baomujicigen & Asteraceae & $\begin{array}{l}\text { Shangri-la, } \\
\text { Weixi and } \\
\text { Deqin }\end{array}$ & Roots & stewed with meat and eaten as tonic. & Jun-Aug & $\begin{array}{l}\text { Fruits, leaves and roots used to } \\
\text { relieve fever, and treat measles, } \\
\text { dysentery and gastropathy. }\end{array}$ & $* * *$ \\
\hline $\begin{array}{l}\text { Arisaema } \\
\text { erubescens (Wall.) } \\
\text { Schott }\end{array}$ & Reduo & Araceae & $\begin{array}{l}\text { Shangri-la, } \\
\text { Weixi and } \\
\text { Deqin }\end{array}$ & $\begin{array}{l}\text { Young } \\
\text { leaves }\end{array}$ & stir-fried & Jun-Jul & $\begin{array}{l}\text { Tubers used to relieve cough } \\
\text { and treat hemoptysis and } \\
\text { pneumonia. }\end{array}$ & ** \\
\hline $\begin{array}{l}\text { Aristolochia } \\
\text { delavayi Franch. }\end{array}$ & Ricaoko & Aristolochiaceae & Shangri-la & $\begin{array}{l}\text { Whole } \\
\text { plants }\end{array}$ & stir-fried and used as spice & Aug-Sept & $\begin{array}{l}\text { Whole plants used as } \\
\text { stomachic tonic. }\end{array}$ & *** \\
\hline $\begin{array}{l}\text { Armeniaca mume } \\
\text { Siebold }\end{array}$ & Kangjue & Rosaceae & $\begin{array}{l}\text { Shangri-la, } \\
\text { Weixi and } \\
\text { Deqin }\end{array}$ & Fruits & eaten fresh. & Aug & $\begin{array}{l}\text { Used as rootstock for } \\
\text { Armeniaca vulgaris. }\end{array}$ & * \\
\hline $\begin{array}{l}\text { Arundinaria faberi } \\
\text { Rendle }\end{array}$ & Sunzi & Poaceae & $\begin{array}{l}\text { Shangri-la, } \\
\text { Weixi and } \\
\text { Deqin }\end{array}$ & New shoots & boiled or stir-fried & Jul-Aug & $\begin{array}{l}\text { Aerial parts used as fodder and } \\
\text { to make bamboo wares. }\end{array}$ & $* * * * *$ \\
\hline $\begin{array}{l}\text { Berberis amoena } \\
\text { Dunn }\end{array}$ & Qiesi & Berberidaceae & $\begin{array}{l}\text { Shangri-la, } \\
\text { Weixi and } \\
\text { Deqin }\end{array}$ & $\begin{array}{l}\text { Young } \\
\text { stems, } \\
\text { leaves and } \\
\text { fruits }\end{array}$ & eaten fresh & May-Sep & $\begin{array}{l}\text { Whole plants used as fence } \\
\text { and hedge plants. }\end{array}$ & *** \\
\hline $\begin{array}{l}\text { Berberis } \\
\text { jamesiana Forrest } \\
\text { et W.W. Sm. }\end{array}$ & Qiesi & Berberidaceae & $\begin{array}{l}\text { Shangri-la, } \\
\text { Weixi and } \\
\text { Deqin }\end{array}$ & $\begin{array}{l}\text { Young } \\
\text { stems, } \\
\text { leaves and } \\
\text { fruits }\end{array}$ & eaten fresh & May-Sep & $\begin{array}{l}\text { Whole plants used as fence } \\
\text { and hedge plants. }\end{array}$ & *** \\
\hline $\begin{array}{l}\text { Berberis pruinosa } \\
\text { Franch. }\end{array}$ & Qiesi & Berberidaceae & $\begin{array}{l}\text { Shangri-la, } \\
\text { Weixi and } \\
\text { Deqin }\end{array}$ & $\begin{array}{l}\text { Young } \\
\text { stems, } \\
\text { leaves and } \\
\text { fruits }\end{array}$ & eaten fresh & May-Sep & $\begin{array}{l}\text { Whole plants used as fence } \\
\text { and hedge plants. }\end{array}$ & $* * *$ \\
\hline $\begin{array}{l}\text { Berberis weisiensis } \\
\text { C. Y. Wu ex S. Y. } \\
\text { Bao }\end{array}$ & Qiesi & Berberidaceae & $\begin{array}{l}\text { Shangri-la, } \\
\text { Weixi and } \\
\text { Deqin }\end{array}$ & $\begin{array}{l}\text { Young } \\
\text { stems, } \\
\text { leaves and } \\
\text { fruits }\end{array}$ & eaten fresh & May-Sep & $\begin{array}{l}\text { Whole plants used as fence } \\
\text { and hedge plants. }\end{array}$ & ** \\
\hline $\begin{array}{l}\text { Berchemia hirtella } \\
\text { Tsai et K. M. Feng }\end{array}$ & Zhila & Rhamnaceae & Deqin & Fruits & eaten fresh & Aug-Sep & & ** \\
\hline $\begin{array}{l}\text { Berchemia hirtella } \\
\text { Tsai et K. M. Feng }\end{array}$ & Zhila & Rhamnaceae & Deqin & $\begin{array}{l}\text { Young } \\
\text { leaves }\end{array}$ & used for making tea & Apr-Jun & & ** \\
\hline $\begin{array}{l}\text { Berchemia sinica } \\
\text { C. K. Schneid. }\end{array}$ & Zhila & Rhamnaceae & Deqin & Fruits & eaten fresh & Aug-Sep & & ** \\
\hline $\begin{array}{l}\text { Berchemia sinica } \\
\text { C. K. Schneid. }\end{array}$ & Zhila & Rhamnaceae & Deqin & $\begin{array}{l}\text { Young } \\
\text { leaves }\end{array}$ & used for making tea & Apr-Jun & & ** \\
\hline $\begin{array}{c}\text { Boehmeria } \\
\text { penduliflora } \\
\text { Wedd. ex Long }\end{array}$ & Sejia & Urticaceae & Deqin & $\begin{array}{l}\text { Young } \\
\text { stems and } \\
\text { leaves }\end{array}$ & boiled or stir-fried & Jun-Jul & & * \\
\hline
\end{tabular}


Table 3 Wild edible plants used by the Tibetans in Shangri-la region, Yunnan Province, China (Continued)

\begin{tabular}{|c|c|c|c|c|c|c|c|c|}
\hline $\begin{array}{l}\text { Boehmeria } \\
\text { tricuspis (Hance) } \\
\text { Makino }\end{array}$ & Sejia & Urticaceae & Deqin & $\begin{array}{l}\text { Young } \\
\text { stems and } \\
\text { leaves }\end{array}$ & stir-fried & Jun-Jul & & * \\
\hline $\begin{array}{l}\text { Broussonetia } \\
\text { papyrifera (L.) } \\
\text { L'Hér. ex Vent. }\end{array}$ & & Moraceae & $\begin{array}{l}\text { Shangri-la, } \\
\text { Weixi and } \\
\text { Deqin }\end{array}$ & Fruits & eaten fresh & Sep-Oct & $\begin{array}{l}\text { Leaves used as fodder. Bark } \\
\text { used for papermaking. }\end{array}$ & * \\
\hline $\begin{array}{l}\text { Capsella bursa- } \\
\text { pastoris (L.) } \\
\text { Medik. }\end{array}$ & Zijisuona & Brassicaceae & $\begin{array}{l}\text { Shangri-la, } \\
\text { Weixi and } \\
\text { Deqin }\end{array}$ & Aerial part & stir-fried & May-Jun & Aerial parts used as fodder. & ** \\
\hline $\begin{array}{l}\text { Cardamine } \\
\text { yunnanensis } \\
\text { Franch. }\end{array}$ & Lijisuona & Brassicaceae & $\begin{array}{l}\text { Shangri-la, } \\
\text { Weixi and } \\
\text { Deqin }\end{array}$ & Aerial part & stir-fried & May-Jun & Aerial parts used as fodder. & ** \\
\hline $\begin{array}{l}\text { Cephalotaxus } \\
\text { fortunei Hook. } \\
\text { var. alpina H. L. Li }\end{array}$ & Miyou & Cephalotaxaceae & Weixi, Deqin & Seeds & eaten fresh or stir-fried & Sep-Oct & $\begin{array}{l}\text { Plants used as fuel-wood. } \\
\text { Seeds used to expel parasite. }\end{array}$ & * \\
\hline $\begin{array}{c}\text { Cerasus } \\
\text { conadenia } \\
\text { (Koehne) T. T. Yu } \\
\text { et C. L. Li }\end{array}$ & Xumumiedu & Rosaceae & $\begin{array}{l}\text { Shangri-la, } \\
\text { Weixi and } \\
\text { Deqin }\end{array}$ & Fruits & eaten fresh & Jul-Aug & $\begin{array}{l}\text { Flowers and leaves used for } \\
\text { weisang }\end{array}$ & $* * *$ \\
\hline $\begin{array}{c}\text { Cerasus } \\
\text { tomentosa } \\
\text { (Thunb.) Wall. }\end{array}$ & Nuosi & Rosaceae & $\begin{array}{l}\text { Shangri-la, } \\
\text { Weixi and } \\
\text { Deqin }\end{array}$ & Fruits & eaten fresh & Jul-Sept & & ** \\
\hline $\begin{array}{l}\text { Chaenomeles } \\
\text { speciosa (Sweet) } \\
\text { Nakai }\end{array}$ & Suomugua & Rosaceae & $\begin{array}{l}\text { Shangri-la, } \\
\text { Weixi and } \\
\text { Deqin }\end{array}$ & Fruits & $\begin{array}{l}\text { stewed with meat as spice and used to prepare local } \\
\text { wine }\end{array}$ & Sept-Oct & & *** \\
\hline $\begin{array}{l}\text { Chenopodium } \\
\text { album L. }\end{array}$ & Hui & Chenopodiaceae & $\begin{array}{l}\text { Shangri-la, } \\
\text { Weixi and } \\
\text { Deqin }\end{array}$ & $\begin{array}{l}\text { Young } \\
\text { stems and } \\
\text { leaves }\end{array}$ & stir-fried & Jun-Jul & Aerial parts used as fodder. & $* * *$ \\
\hline $\begin{array}{l}\text { Cinnamomum } \\
\text { glanduliferum } \\
\text { (Wall.) Meisner }\end{array}$ & Xiangzhangzi & Lauraceae & $\begin{array}{l}\text { Shangri-la, } \\
\text { Weixi and } \\
\text { Deqin }\end{array}$ & Fruits & stir-fried and used as spices & Aug-Sept & $\begin{array}{l}\text { Fruits used to treat } \\
\text { stomachache. }\end{array}$ & * \\
\hline $\begin{array}{c}\text { Cirsium } \\
\text { japonicum } \\
\text { (Thunb.) Fisch. ex } \\
\text { DC. }\end{array}$ & Baimaci & Asteraceae & $\begin{array}{l}\text { Shangri-la, } \\
\text { Weixi and } \\
\text { Deqin }\end{array}$ & Roots & stewed with meat and eaten as tonic & Jun-Aug & $\begin{array}{l}\text { Young stems and leaves used } \\
\text { as fodder. }\end{array}$ & $* * *$ \\
\hline $\begin{array}{c}\text { Codonopsis } \\
\text { pilosula (Franch.) } \\
\text { Nannf. var. } \\
\text { handeliana } \\
\text { (Nannf.) L. T. } \\
\text { Shen }\end{array}$ & Dangshen & Campanulaceae & $\begin{array}{l}\text { Deqin and } \\
\text { Weixi }\end{array}$ & Roots & stewed with meat and eaten as tonic & Jul-Sept & $\begin{array}{l}\text { Aerial parts used as fodder. } \\
\text { Roots used to invigorate the } \\
\text { spleen. }\end{array}$ & $* * *$ \\
\hline $\begin{array}{c}\text { Coriaria } \\
\text { nepalensis Wall. }\end{array}$ & Masen & Coriariaceae & Weixi & Fruits & eaten fresh & May-Jun & & * \\
\hline
\end{tabular}


Table 3 Wild edible plants used by the Tibetans in Shangri-la region, Yunnan Province, China (Continued)

\begin{tabular}{|c|c|c|c|c|c|c|c|c|}
\hline $\begin{array}{c}\text { Cornus capitata } \\
\text { Wall. }\end{array}$ & Jisuo; Jisuziguo & Cornaceae & $\begin{array}{l}\text { Shangri-la, } \\
\text { Weixi and } \\
\text { Deqin }\end{array}$ & Fruits & eaten fresh & Aug-Sept & $\begin{array}{l}\text { Fruits, stems and leaves used } \\
\text { as veterinary medicine. }\end{array}$ & $* * *$ \\
\hline $\begin{array}{l}\text { Cornus } \\
\text { macrophylla Wall. }\end{array}$ & Dengtaishu & Cornaceae & $\begin{array}{l}\text { Shangri-la, } \\
\text { Weixi and } \\
\text { Deqin }\end{array}$ & Seeds & used for making vegetable oil. & Aug-Sept & Plants used as fuel-wood. & $* *$ \\
\hline $\begin{array}{l}\text { Cornus schindleri } \\
\text { Wangerin }\end{array}$ & Saisaizi & Cornaceae & $\begin{array}{l}\text { Shangri-la, } \\
\text { Weixi and } \\
\text { Deqin }\end{array}$ & seeds & used for making vegetable oil & Aug-Sept & Plants used as fuel-wood. & $* *$ \\
\hline $\begin{array}{l}\text { Cornus ulotricha } \\
\text { C. K. Schneid. et } \\
\text { Wangerin }\end{array}$ & Dengtaishu & Cornaceae & $\begin{array}{l}\text { Shangri-la, } \\
\text { Weixi and } \\
\text { Deqin }\end{array}$ & seeds & used for making vegetable oil & Aug-Sept & Plants used as fuel-wood. & * \\
\hline $\begin{array}{l}\text { Corylus chinensis } \\
\text { Franch. }\end{array}$ & Jilizi & Betulaceae & $\begin{array}{l}\text { Shangri-la, } \\
\text { Weixi and } \\
\text { Deqin }\end{array}$ & Fruits & used for making pastries & Sept-Oct & $\begin{array}{l}\text { Wood used for construction or } \\
\text { furniture. }\end{array}$ & $* *$ \\
\hline $\begin{array}{c}\text { Conylus } \\
\text { yunnanensis } \\
\text { (Franch.) Camus }\end{array}$ & Shanbaiguo & Betulaceae & $\begin{array}{l}\text { Shangri-la, } \\
\text { Weixi and } \\
\text { Deqin }\end{array}$ & Fruits & used for making pastries & Sept-Oct & $\begin{array}{l}\text { Woods used for construction } \\
\text { or furniture. }\end{array}$ & * \\
\hline $\begin{array}{c}\text { Cotinus coggygria } \\
\text { Scop. var. } \\
\text { glaucophylla C. Y. } \\
\text { Wu }\end{array}$ & Jiade & Anacardiaceae & Shangri-la & $\begin{array}{l}\text { Young } \\
\text { leaves }\end{array}$ & boiled or stir-fried & May-Jun & $\begin{array}{l}\text { Whole plants used as } \\
\text { ornamental. }\end{array}$ & * \\
\hline $\begin{array}{c}\text { Crataegus } \\
\text { chungtienensis W. } \\
\text { W. Sm. }\end{array}$ & Lubu & Rosaceae & $\begin{array}{l}\text { Weixi and } \\
\text { Shangri-la }\end{array}$ & Fruits & eaten fresh & Sept & $\begin{array}{l}\text { Whole plants used as fence } \\
\text { and hedge plants. }\end{array}$ & $* * *$ \\
\hline $\begin{array}{l}\text { Crataegus oresbia } \\
\text { W. W. Sm. }\end{array}$ & Lubu & Rosaceae & $\begin{array}{l}\text { Weixi and } \\
\text { Shangri-la }\end{array}$ & Fruits & eaten fresh & Aug-Sept & $\begin{array}{l}\text { Whole plants used as fence } \\
\text { and hedge plants. }\end{array}$ & $* * *$ \\
\hline $\begin{array}{l}\text { Cynanchum } \\
\text { forrestii Schltr. }\end{array}$ & Babeda & Asclepiadaceae & $\begin{array}{l}\text { Deqin and } \\
\text { Weixi }\end{array}$ & Fruits & eaten fresh & Aug-Oct & $\begin{array}{l}\text { Roots stewed with meat and } \\
\text { eaten to treat rheumatism. }\end{array}$ & * \\
\hline $\begin{array}{l}\text { Davidia } \\
\text { involucrata Baill. } \\
\text { var. vilmoriniana } \\
\text { (Dode) Wangerin }\end{array}$ & Labizi & Nyssaceae & Weixi & Fruits & eaten fresh & Sept-Oct & $\begin{array}{l}\text { Whole plant used as } \\
\text { ornamental. }\end{array}$ & * \\
\hline $\begin{array}{l}\text { Debregeasia } \\
\text { orientalis C. J. } \\
\text { Chen }\end{array}$ & Jiaojia & Urticaceae & $\begin{array}{l}\text { Shangri-la, } \\
\text { Weixi and } \\
\text { Deqin }\end{array}$ & Fruits & eaten fresh and used to make local wine & Jun-Aug & $\begin{array}{l}\text { Roots used to treat rheumatoid } \\
\text { arthritis and broken bones. }\end{array}$ & * \\
\hline $\begin{array}{c}\text { Decaisnea insignis } \\
\text { (Griff.) Hook. f. et } \\
\text { Thomson }\end{array}$ & Xianli & Lardizabalaceae & $\begin{array}{l}\text { Shangri-la, } \\
\text { Weixi and } \\
\text { Deqin }\end{array}$ & Fruits & eaten fresh and used to make local wine & Jul-Aug & $\begin{array}{l}\text { Roots and fruits used to } \\
\text { clearing heat. }\end{array}$ & *** \\
\hline $\begin{array}{c}\text { Dioscorea } \\
\text { melanophyma } \\
\text { Prain et Burkill }\end{array}$ & Huangshayue & Dioscoreaceae & Weixi & Tubers & boiled or stir-fried & Jun-Jul & Aerial parts used as fodder. & $* *$ \\
\hline Diospyros lotus L. & Tazhi & Ebenaceae & $\begin{array}{l}\text { Shangri-la, } \\
\text { Weixi and } \\
\text { Deqin }\end{array}$ & Fruits & eaten fresh & Sept-Oct & & *** \\
\hline
\end{tabular}


Table 3 Wild edible plants used by the Tibetans in Shangri-la region, Yunnan Province, China (Continued)

\begin{tabular}{|c|c|c|c|c|c|c|c|c|}
\hline $\begin{array}{l}\text { Duchesnea indica } \\
\text { (Andrews) Focke }\end{array}$ & Dihongpao & Rosaceae & $\begin{array}{l}\text { Shangri-la, } \\
\text { Weixi and } \\
\text { Deqin }\end{array}$ & Fruits & eaten fresh & Jun-Jul & & * \\
\hline $\begin{array}{l}\text { Elaeagnus } \\
\text { multiflora Thunb. }\end{array}$ & Cibie & Elaeagnaceae & $\begin{array}{l}\text { Shangri-la, } \\
\text { Weixi and } \\
\text { Deqin }\end{array}$ & Fruits & eaten fresh & Jun-Jul & & $* *$ \\
\hline $\begin{array}{l}\text { Elaeagnus } \\
\text { umbellata Thunb. }\end{array}$ & Yangnaiguo & Elaeagnaceae & $\begin{array}{l}\text { Shangri-la, } \\
\text { Weixi and } \\
\text { Deqin }\end{array}$ & Fruits & eaten fresh & Jul-Aug & & $* *$ \\
\hline $\begin{array}{l}\text { Eriobotrya } \\
\text { salwinensis } \\
\text { Hand.-Mazz. }\end{array}$ & & Rosaceae & $\begin{array}{l}\text { Weixi and } \\
\text { Deqin }\end{array}$ & Fruits & eaten fresh & Jun-Aug & Plants used as fuel-wood. & $*$ \\
\hline $\begin{array}{c}\text { Eutrema } \\
\text { deltoideum } \\
\text { (Hook. f. et } \\
\text { Thomson) O. E. } \\
\text { Schulz } \\
\end{array}$ & Limo & Brassicaceae & $\begin{array}{l}\text { Shangri-la, } \\
\text { Weixi and } \\
\text { Deqin }\end{array}$ & $\begin{array}{l}\text { Young } \\
\text { stems and } \\
\text { leaves }\end{array}$ & stir-fried & May-Jun & Aerial parts used as fodder. & ** \\
\hline $\begin{array}{l}\text { Eutrema } \\
\text { heterophyllum (W. } \\
\text { W. Sm.) H. Hara }\end{array}$ & Limo & Brassicaceae & $\begin{array}{l}\text { Shangri-la, } \\
\text { Weixi and } \\
\text { Deqin }\end{array}$ & $\begin{array}{l}\text { Young } \\
\text { stems and } \\
\text { leaves }\end{array}$ & stir-fried & May-Jun & Aerial parts used as fodder. & $* *$ \\
\hline $\begin{array}{l}\text { Eutrema } \\
\text { himalaicum } \\
\text { Hook. f. et } \\
\text { Thomson }\end{array}$ & Limo & Brassicaceae & $\begin{array}{l}\text { Shangri-la, } \\
\text { Weixi and } \\
\text { Deqin }\end{array}$ & $\begin{array}{l}\text { Young } \\
\text { stems and } \\
\text { leaves }\end{array}$ & stir-fried & May-Jun & Aerial parts used as fodder. & ** \\
\hline $\begin{array}{c}\text { Fagopyrum } \\
\text { dibotrys (D. Don) } \\
\text { H. Hara }\end{array}$ & Wanao & Polygonaceae & Deqin & $\begin{array}{l}\text { Young } \\
\text { stems and } \\
\text { leaves }\end{array}$ & stir-fried & Jun-Aug & Aerial parts used as fodder. & $*$ \\
\hline $\begin{array}{c}\text { Fargesia } \\
\text { melanostachys } \\
\text { (Hand.-Mazz.) T. } \\
\text { P. Yi } \\
\end{array}$ & Sunzi & Poaceae & $\begin{array}{l}\text { Shangri-la, } \\
\text { Weixi and } \\
\text { Deqin }\end{array}$ & New shoots & boiled or stir-fried & May-Aug & $\begin{array}{l}\text { Aerial parts used as fodder and } \\
\text { to make bamboo wares. }\end{array}$ & $* * * * *$ \\
\hline Ficus pumila L. & Dongshili & Moraceae & $\begin{array}{l}\text { Shangri-la, } \\
\text { Weixi and } \\
\text { Deqin }\end{array}$ & Fruits & used for making bean jelly & Jul-Aug & Leaves used as fodder. & $*$ \\
\hline $\begin{array}{l}\text { Ficus sarmentosa } \\
\text { Buch.-Ham. ex } \\
\text { Sm. }\end{array}$ & dongshili & Moraceae & $\begin{array}{l}\text { Shangri-la, } \\
\text { Weixi and } \\
\text { Deqin }\end{array}$ & Fruits & used for making bean jelly & Jul-Aug & Leaves used as fodder. & $*$ \\
\hline $\begin{array}{l}\text { Foeniculum } \\
\text { vulgare Mill. }\end{array}$ & Asi & Apiaceae & $\begin{array}{l}\text { Shangri-la, } \\
\text { Weixi and } \\
\text { Deqin }\end{array}$ & $\begin{array}{l}\text { Young } \\
\text { stems and } \\
\text { leaves }\end{array}$ & eaten fresh or stir-fried & May-Jul & & $* *$ \\
\hline $\begin{array}{c}\text { Fragaria } \\
\text { moupinensis } \\
\text { (Franch.) Cardot }\end{array}$ & Gasuo & Rosaceae & $\begin{array}{l}\text { Shangri-la, } \\
\text { Weixi and } \\
\text { Deqin }\end{array}$ & Fruits & eaten fresh. & Jun-Jul & Whole plants used as fodder. & $* *$ \\
\hline $\begin{array}{l}\text { Galinsoga } \\
\text { parviflora Cav. }\end{array}$ & Nawabijia & Asteraceae & $\begin{array}{l}\text { Deqin and } \\
\text { Weixi }\end{array}$ & $\begin{array}{l}\text { Young } \\
\text { stems and } \\
\text { leaves }\end{array}$ & boiled or stir-fried & Jun-Aug & Whole plants used as fodder. & $*$ \\
\hline
\end{tabular}


Table 3 Wild edible plants used by the Tibetans in Shangri-la region, Yunnan Province, China (Continued)

\begin{tabular}{|c|c|c|c|c|c|c|c|c|}
\hline Ginkgo biloba L. & Baiguo & Ginkgoaceae & Deqin, Weixi & Seeds & eaten fresh or stir-fried & Sep-Oct & Seeds used to treat asthma. & * \\
\hline $\begin{array}{l}\text { Gnaphalium } \\
\text { affine D. Don }\end{array}$ & Qingmincai & Asteraceae & Weixi & $\begin{array}{l}\text { Young } \\
\text { leaves }\end{array}$ & grounded with sticky rice to make rice cake. & Apr-May & $\begin{array}{l}\text { Leaves used to treat cuts and } \\
\text { gun shot wounds. }\end{array}$ & * \\
\hline $\begin{array}{l}\text { Herminium } \\
\text { lanceum (Thunb. } \\
\text { ex Sw.) Vuijk }\end{array}$ & Lianxiongde & Orchidaceae & Shangri-la & Whole plant & stewed with meat and eaten as tonic. & Aug-Sep & Whole plant used as fodder. & * \\
\hline $\begin{array}{c}\text { Hippophae } \\
\text { rhamnoides } \mathrm{L} \text {. } \\
\text { subsp. } \\
\text { yunnanensis } \\
\text { Rousi }\end{array}$ & Xiju & Elaeagnaceae & $\begin{array}{l}\text { Deqin. } \\
\text { Shangri-la }\end{array}$ & Fruits & eaten fresh or used to make beverage and wine. & Aug-Oct & $\begin{array}{l}\text { Fruits used to treat cough and } \\
\text { invigorate the circulation of } \\
\text { blood. }\end{array}$ & $* * * * *$ \\
\hline $\begin{array}{c}\text { Houttuynia } \\
\text { cordata Thunb. }\end{array}$ & Zhergen & Saururaceae & $\begin{array}{l}\text { Weixi, } \\
\text { Shangri-la }\end{array}$ & $\begin{array}{l}\text { Leaves and } \\
\text { roots }\end{array}$ & eaten fresh or stir-fried & Jun-Jul & & $* * *$ \\
\hline Juglans regia L. & Daiga & Juglandaceae & $\begin{array}{l}\text { Shangri-la, } \\
\text { Weixi and } \\
\text { Deqin }\end{array}$ & Seeds & $\begin{array}{l}\text { eaten fresh or stir-fried, and used for making vegetable } \\
\text { oil. }\end{array}$ & Aug-Sept & Plants used as fuel-wood. & $* * *$ \\
\hline $\begin{array}{c}\text { Kalopanax } \\
\text { septemlobus } \\
\text { (Thunb.) Koidz. }\end{array}$ & Cilaobao & Araliaceae & $\begin{array}{l}\text { Shangri-la, } \\
\text { Weixi and } \\
\text { Deqin }\end{array}$ & $\begin{array}{l}\text { Young } \\
\text { stems and } \\
\text { leaves }\end{array}$ & eaten fresh or stir-fried & May-Jun & & ** \\
\hline $\begin{array}{l}\text { Lethariella } \\
\text { cladonioides (Nyl.) } \\
\text { Krog }\end{array}$ & Gangge & Parmeliaceae & Deqin & Whole plant & used for making tea, wine and beverage & Aug-Oct & $\begin{array}{l}\text { Used to tranquilize mind and } \\
\text { clearing heat. }\end{array}$ & * \\
\hline $\begin{array}{l}\text { Leycesteria } \\
\text { formosa Wall. }\end{array}$ & Sezha & Caprifoliaceae & Deqin & Fruits & eaten fresh. & Aug-Oct & & * \\
\hline $\begin{array}{c}\text { Ligusticum } \\
\text { daucoides } \\
\text { (Franch.) Franch. }\end{array}$ & Riqincai & Apiaceae & Shangri-la & $\begin{array}{l}\text { Whole } \\
\text { plants }\end{array}$ & stir-fried or added to soups & Apr-May & Aerial parts used as fodder. & $* * * *$ \\
\hline $\begin{array}{l}\text { Lindera kariensis } \\
\text { W. W. Sm. }\end{array}$ & Rihujiao & Lauraceae & Weixi, Deqin & Fruits & used as spices & Jul-Sept & & ** \\
\hline $\begin{array}{l}\text { Lindera nacusua } \\
\text { (D. Don) Merr. }\end{array}$ & Rihujiao & Lauraceae & Weixi & Fruits & used as spices & Jul-Sept & & ** \\
\hline $\begin{array}{c}\text { Lindera } \\
\text { obtusiloba Blume } \\
\text { var. heterophylla } \\
\text { (Meisn.) H. P. Tsui } \\
\end{array}$ & Rihujiao & Lauraceae & Weixi & Fruits & used as spices & Jul-Sept & & * \\
\hline $\begin{array}{l}\text { Lindera reflexa } \\
\text { Hemsl. }\end{array}$ & Rihujiao & Lauraceae & Weixi & Fruits & used as spices & Jul-Sept & & * \\
\hline Lobaria sp. & Qingwapi & Stictaceae & $\begin{array}{l}\text { Shangri-la } \\
\text { and Weixi }\end{array}$ & Aerial part & eaten fresh & Jul-Sept & $\begin{array}{l}\text { Whole plant used to treat } \\
\text { dyspepsia. }\end{array}$ & * \\
\hline $\begin{array}{l}\text { Lycopus lucidus } \\
\text { Turcz. ex Benth. }\end{array}$ & Ganluo & Lamiaceae & Shangri-la & $\begin{array}{l}\text { Young } \\
\text { stems and } \\
\text { leaves }\end{array}$ & eaten fresh or stir-fried or used for making pickle & Jul-Aug & & ** \\
\hline
\end{tabular}


Table 3 Wild edible plants used by the Tibetans in Shangri-la region, Yunnan Province, China (Continued)

\begin{tabular}{|c|c|c|c|c|c|c|c|c|}
\hline $\begin{array}{l}\text { Mahonia } \\
\text { duclouxiana } \\
\text { Gagnep. }\end{array}$ & Jisa & Berberidaceae & Deqin & Fruits & eaten fresh. & Aug-Sep & $\begin{array}{l}\text { Whole plants used as hedge } \\
\text { plants. }\end{array}$ & * \\
\hline $\begin{array}{l}\text { Maianthemum } \\
\text { atropurpureum } \\
\text { (Franch.) } \\
\text { LaFrankie }\end{array}$ & Zhuyecai;Nibai & Liliaceae & $\begin{array}{l}\text { Shangri-la, } \\
\text { Weixi and } \\
\text { Deqin }\end{array}$ & $\begin{array}{l}\text { Young } \\
\text { shoots and } \\
\text { leaves }\end{array}$ & stir-fried or added to soups & May-Jun & Aerial parts used as fodder. & $* * * * *$ \\
\hline $\begin{array}{l}\text { Maianthemum } \\
\text { forrestii (W. W. } \\
\text { Smith) LaFrankie }\end{array}$ & Zhuyecai;Nibai & Liliaceae & $\begin{array}{l}\text { Shangri-la } \\
\text { and Weixi }\end{array}$ & $\begin{array}{l}\text { Young } \\
\text { shoots and } \\
\text { leaves }\end{array}$ & stir-fried or added to soups & May-Jun & Aerial parts used as fodder. & $* * * *$ \\
\hline $\begin{array}{l}\text { Maianthemum } \\
\text { henryi (Baker) } \\
\text { LaFrankie }\end{array}$ & Zhuyecai;Nibai & Liliaceae & $\begin{array}{l}\text { Shangri-la, } \\
\text { Weixi and } \\
\text { Deqin }\end{array}$ & $\begin{array}{l}\text { Young } \\
\text { shoots and } \\
\text { leaves }\end{array}$ & stir-fried or added to soups & May-Jun & Aerial parts used as fodder. & $* * * *$ \\
\hline $\begin{array}{l}\text { Maianthemum } \\
\text { oleraceum (Baker) } \\
\text { LaFrankie }\end{array}$ & Zhuyecai;Nibai & Liliaceae & $\begin{array}{l}\text { Weixi, } \\
\text { Shangri-la }\end{array}$ & $\begin{array}{l}\text { Young } \\
\text { shoots and } \\
\text { leaves }\end{array}$ & stir-fried or added to soups & May-Jun & Aerial parts used as fodder. & $* * *$ \\
\hline $\begin{array}{l}\text { Maianthemum } \\
\text { purpureum } \\
\text { (Wallich) } \\
\text { LaFrankie }\end{array}$ & Zhuyecai;Nibai & Liliaceae & $\begin{array}{l}\text { Shangri-la, } \\
\text { Weixi and } \\
\text { Deqin }\end{array}$ & $\begin{array}{l}\text { Young } \\
\text { shoots and } \\
\text { leaves }\end{array}$ & stir-fried or added to soups & May-Jun & Aerial parts used as fodder. & $* * * * *$ \\
\hline $\begin{array}{c}\text { Maianthemum } \\
\text { tatsienense } \\
\text { (Franct.) } \\
\text { LaFrankie }\end{array}$ & Zhuyecai;Nibai & Liliaceae & Shangri-la & $\begin{array}{l}\text { Young } \\
\text { shoots and } \\
\text { leaves }\end{array}$ & stir-fried or added to soups & May-Jun & Aerial parts used as fodder. & $* * * *$ \\
\hline $\begin{array}{l}\text { Malus rockii } \\
\text { Rehder }\end{array}$ & Tangli & Rosaceae & $\begin{array}{l}\text { Shangri-la, } \\
\text { Weixi and } \\
\text { Deqin }\end{array}$ & Fruits & eaten fresh & Sept & $\begin{array}{l}\text { Plants used as fuel-wood, and } \\
\text { rootstock for Malus pumila. } \\
\text { Whole plants used as fence. }\end{array}$ & $* * *$ \\
\hline $\begin{array}{l}\text { Malus spectabilis } \\
\text { (Ait.) Borkh. }\end{array}$ & Haitangguo & Rosaceae & $\begin{array}{l}\text { Shangri-la, } \\
\text { Weixi and } \\
\text { Deqin }\end{array}$ & Fruits & eaten fresh & Aug-Sept & $\begin{array}{l}\text { Fruits decoction used to treat } \\
\text { dark urine. }\end{array}$ & ** \\
\hline $\begin{array}{l}\text { Malva verticillata } \\
\mathrm{L} .\end{array}$ & Jiangba & Malvaceae & Deqin & $\begin{array}{l}\text { Young } \\
\text { stems and } \\
\text { leaves }\end{array}$ & stir-fried & Jun-Aug & $\begin{array}{l}\text { Leaves, stems and seeds used } \\
\text { as fodder. Whole plant used as } \\
\text { ornamental. }\end{array}$ & * \\
\hline $\begin{array}{c}\text { Matteuccia } \\
\text { struthiopteris (L.) } \\
\text { Tadaro } \\
\end{array}$ & Huangguaxiang & Onocleaceae & $\begin{array}{l}\text { Shangri-la, } \\
\text { Weixi and } \\
\text { Deqin }\end{array}$ & $\begin{array}{l}\text { Immature } \\
\text { fronds }\end{array}$ & eaten fresh or stir-fried & May-Jun & & $* * * *$ \\
\hline $\begin{array}{l}\text { Medicago } \\
\text { lupulina L. }\end{array}$ & Mocuo & Fabaceae & $\begin{array}{l}\text { Deqin, } \\
\text { Shangri-la }\end{array}$ & $\begin{array}{l}\text { Young } \\
\text { stems and } \\
\text { leaves }\end{array}$ & eaten fresh or stir-fried & Jun-Jul & $\begin{array}{l}\text { Leaves, stems, flowers and } \\
\text { seeds used as fodder. }\end{array}$ & * \\
\hline $\begin{array}{l}\text { Megacarpaea } \\
\text { delavayi Franch. }\end{array}$ & Yuose & Brassicaceae & $\begin{array}{l}\text { Shangri-la, } \\
\text { Weixi and } \\
\text { Deqin }\end{array}$ & $\begin{array}{l}\text { Young } \\
\text { stems and } \\
\text { leaves }\end{array}$ & stir-fried & May-Jun & Aerial parts used as fodder. & ** \\
\hline $\begin{array}{l}\text { Megacarpaea } \\
\text { polyandra Benth. } \\
\text { ex Madden }\end{array}$ & Yuose & Brassicaceae & $\begin{array}{l}\text { Shangri-la, } \\
\text { Weixi and } \\
\text { Deqin }\end{array}$ & $\begin{array}{l}\text { Young } \\
\text { stems and } \\
\text { leaves }\end{array}$ & stir-fried & May-Jun & Aerial parts used as fodder. & ** \\
\hline
\end{tabular}


Table 3 Wild edible plants used by the Tibetans in Shangri-la region, Yunnan Province, China (Continued)

\begin{tabular}{|c|c|c|c|c|c|c|c|c|}
\hline $\begin{array}{c}\text { Mentha } \\
\text { canadensis L. }\end{array}$ & Qiubi & Lamiaceae & $\begin{array}{l}\text { Shangri-la, } \\
\text { Weixi and } \\
\text { Deqin }\end{array}$ & $\begin{array}{l}\text { Young } \\
\text { leaves }\end{array}$ & eaten fresh or stir-fried & Jun-Aug & & $* *$ \\
\hline $\begin{array}{l}\text { Nostoc } \\
\text { sphaerioides } \\
\text { Kützing }\end{array}$ & Shuimuer & Nostocaceae & Shangri-la & Whole plant & eaten fresh or added to soups & Jun-Jul & $\begin{array}{l}\text { Whole plant used to treat } \\
\text { burns and scalds. }\end{array}$ & * \\
\hline $\begin{array}{l}\text { Metapanax } \\
\text { delavayi (Franch.) } \\
\text { J. Wen et Frodin }\end{array}$ & & Araliaceae & Deqin, Weixi & $\begin{array}{l}\text { Young } \\
\text { leaves }\end{array}$ & used for making tea & Apr-May & $\begin{array}{l}\text { Whole plants used as hedge } \\
\text { plants. }\end{array}$ & * \\
\hline $\begin{array}{l}\text { Ophioglossum } \\
\text { reticulatum } \mathrm{L} \text {. }\end{array}$ & Yimuyidun & Ophioglossaceae & Shangri-la & $\begin{array}{l}\text { Immature } \\
\text { fronds }\end{array}$ & stir-fried or added to soups & Jul-Aug & $\begin{array}{l}\text { Whole plants used to treat } \\
\text { impotence and lumbago. }\end{array}$ & * \\
\hline $\begin{array}{l}\text { Opuntia } \\
\text { monacantha } \\
\text { (Willd.) Haw. }\end{array}$ & Xianrenguo & Cactaceae & $\begin{array}{l}\text { Shangri-la, } \\
\text { Weixi and } \\
\text { Deqin }\end{array}$ & Fruits & eaten fresh & Aug-Sep & $\begin{array}{l}\text { Tubers and fruits used as } \\
\text { fodder. Whole plants used as } \\
\text { fence and hedge plants. }\end{array}$ & *** \\
\hline $\begin{array}{l}\text { Oreorchis indica } \\
\text { (Lindl.) Hook. f. }\end{array}$ & Xiabaji & Orchidaceae & $\begin{array}{l}\text { Shangri-la, } \\
\text { Weixi and } \\
\text { Deqin }\end{array}$ & Pseudobulbs & boiled or stir-fried & Jun-Aug & $\begin{array}{l}\text { Whole plants used as fodder. } \\
\text { Pseudobulbs used to stop } \\
\text { bleeding and detumescence. }\end{array}$ & * \\
\hline $\begin{array}{c}\text { Osmunda } \\
\text { japonica Thunb. }\end{array}$ & Shuijuecai & Osmundaceae & Weixi & $\begin{array}{l}\text { Immature } \\
\text { fronds }\end{array}$ & stir-fried & May-Jun & & *** \\
\hline $\begin{array}{l}\text { Osteomeles } \\
\text { schwerinae C. K. } \\
\text { Schneid. }\end{array}$ & Sele & Rosaceae & $\begin{array}{l}\text { Shangri-la, } \\
\text { Weixi and } \\
\text { Deqin }\end{array}$ & Fruits & eaten fresh & Aug-Sept & $\begin{array}{l}\text { Leaves and fruits used as } \\
\text { fodder. }\end{array}$ & $* *$ \\
\hline $\begin{array}{l}\text { Panax japonicus } \\
\text { (T. Nees) C. A. } \\
\text { Meyer var. major } \\
\text { (Burkill) C. Y. Wu } \\
\text { et K. M. Feng }\end{array}$ & Gedeqi & Araliaceae & Shangri-la & $\begin{array}{l}\text { Young } \\
\text { stems and } \\
\text { leaves }\end{array}$ & eaten fresh or stir-fried & May-Jun & $\begin{array}{l}\text { Whole plants used as fodder. } \\
\text { Roots used to stop bleeding. }\end{array}$ & *** \\
\hline $\begin{array}{l}\text { Panax japonicus } \\
\text { (T. Nees) C. A. } \\
\text { Meyer var. major } \\
\text { (Burkill) C. Y. Wu } \\
\text { et K. M. Feng }\end{array}$ & Gedeqi & Araliaceae & Shangri-la & Rhizomes & stewed with meat and eaten as tonic. & Jul-Aug & $\begin{array}{l}\text { Whole plants used as fodder. } \\
\text { Rhizomes used to stop } \\
\text { bleeding. }\end{array}$ & *** \\
\hline $\begin{array}{l}\text { Pentapanax } \\
\text { henryi Harms }\end{array}$ & & Araliaceae & $\begin{array}{l}\text { Shangri-la, } \\
\text { Weixi and } \\
\text { Deqin }\end{array}$ & $\begin{array}{l}\text { Young } \\
\text { stems and } \\
\text { leaves }\end{array}$ & eaten fresh or stir-fried & Apr-May & & $* *$ \\
\hline $\begin{array}{c}\text { Photinia } \\
\text { glomerata } \\
\text { Rehder et E. H. } \\
\text { Wilson }\end{array}$ & Chongsi & Rosaceae & Deqin & Fruits & eaten fresh & Sept & & * \\
\hline $\begin{array}{l}\text { Phyllanthus } \\
\text { emblica L. }\end{array}$ & Ganlan & Euphorbiaceae & Shangri-la & Fruits & eaten fresh & Jul-Sept & Barks used to extract tannin. & $* * *$ \\
\hline $\begin{array}{l}\text { Phytolacca } \\
\text { acinosa Roxb. }\end{array}$ & Tuogiong & Phytolaccaceae & Deqin & $\begin{array}{l}\text { Young } \\
\text { stems and } \\
\text { leaves }\end{array}$ & eaten fresh or stir-fried & Jul-Aug & $\begin{array}{l}\text { Roots used to promote } \\
\text { diuresis. }\end{array}$ & * \\
\hline
\end{tabular}


Table 3 Wild edible plants used by the Tibetans in Shangri-la region, Yunnan Province, China (Continued)

\begin{tabular}{|c|c|c|c|c|c|c|c|c|}
\hline $\begin{array}{l}\text { Pinellia } \\
\text { pedatisecta } \\
\text { Schott }\end{array}$ & Luoa & Araceae & Deqin & $\begin{array}{l}\text { Young } \\
\text { leaves }\end{array}$ & stir-fried & Jun-Jul & $\begin{array}{l}\text { Corms used to treat vomit and } \\
\text { reduce phlegm. }\end{array}$ & * \\
\hline $\begin{array}{l}\text { Pinus armandii } \\
\text { Franch. }\end{array}$ & Seitu; Songzi & Pinaceae & $\begin{array}{l}\text { Shangri-la, } \\
\text { Weixi and } \\
\text { Deqin }\end{array}$ & Seeds & eaten fresh or stir-fried & Sept-Oct & $\begin{array}{l}\text { Leaves and stems used for } \\
\text { weisang. Needles used as } \\
\text { fodder. Plants used as fuel- } \\
\text { wood. }\end{array}$ & ** \\
\hline $\begin{array}{c}\text { Pistacia } \\
\text { weinmanniifolia J. } \\
\text { Poiss. ex Franch. }\end{array}$ & $\mathrm{Li}$ & Anacardiaceae & Deqin & Fruits & eaten fresh & Aug-Sept & $\begin{array}{l}\text { Leaves and stems used for } \\
\text { weisang. Leaves and fruits used } \\
\text { as fodder. }\end{array}$ & $* * * *$ \\
\hline $\begin{array}{c}\text { Plantago asiatica } \\
\text { L. }\end{array}$ & Hamaye & Plantaginaceae & $\begin{array}{l}\text { Shangri-la, } \\
\text { Weixi and } \\
\text { Deqin }\end{array}$ & $\begin{array}{l}\text { Whole } \\
\text { plants }\end{array}$ & boiled or stir-fried & Jun-Aug & $\begin{array}{l}\text { Leaves, stems, flowers and } \\
\text { seeds used as fodder. }\end{array}$ & $* * *$ \\
\hline Plantago major L. & Hamaye & Plantaginaceae & $\begin{array}{l}\text { Shangri-la, } \\
\text { Weixi and } \\
\text { Deqin }\end{array}$ & $\begin{array}{l}\text { Whole } \\
\text { plants }\end{array}$ & boiled or stir-fried & Jun-Aug & $\begin{array}{l}\text { Leaves, stems, flowers and } \\
\text { seeds used as fodder. }\end{array}$ & $* * *$ \\
\hline $\begin{array}{l}\text { Potentilla } \\
\text { anserina } \mathrm{L} .\end{array}$ & Chuomo & Rosaceae & $\begin{array}{l}\text { Shangri-la, } \\
\text { Weixi and } \\
\text { Deqin }\end{array}$ & Roots & eaten fresh or boiled & Jun-Sept & $\begin{array}{l}\text { Leaves, stems and fruits used } \\
\text { as fodder. Roots used to } \\
\text { control leukorrhea flow. }\end{array}$ & $* * *$ \\
\hline $\begin{array}{l}\text { Potentilla } \\
\text { coriandrifolia D. } \\
\text { Don var. dumosa } \\
\text { Franch. } \\
\end{array}$ & Zumuyasha & Rosaceae & $\begin{array}{l}\text { Shangri-la, } \\
\text { Weixi and } \\
\text { Deqin }\end{array}$ & Roots & eaten after boiling & Jun-Sept & Whole plants used as fodder. & $*$ \\
\hline $\begin{array}{l}\text { Potentilla } \\
\text { leuconota D. Don }\end{array}$ & Pagu & Rosaceae & $\begin{array}{l}\text { Shangri-la, } \\
\text { Weixi and } \\
\text { Deqin }\end{array}$ & Roots & eaten after boiling & Jun-Sept & Whole plants used as fodder. & * \\
\hline $\begin{array}{c}\text { Prasiola } \\
\text { subareolata Skuja. }\end{array}$ & Shihuacai & Prasiolaceae & Shangri-la & $\begin{array}{l}\text { Whole } \\
\text { plants }\end{array}$ & eaten fresh or added to soups & Jun-Jul & & $*$ \\
\hline $\begin{array}{l}\text { Prinsepia utilis } \\
\text { Royle }\end{array}$ & Qingciguo & Rosaceae & $\begin{array}{l}\text { Shangri-la, } \\
\text { Weixi and } \\
\text { Deqin }\end{array}$ & Seeds & used for making vegetable oil & Jul-Aug & & $* * *$ \\
\hline $\begin{array}{c}\text { Pteridium } \\
\text { aquilinum (L.) } \\
\text { Kuhn var. } \\
\text { latiusclum (Desv.) } \\
\text { Underw. ex A. } \\
\text { Heller }\end{array}$ & Zhila & Pteridaceae & $\begin{array}{l}\text { Shangri-la, } \\
\text { Weixi and } \\
\text { Deqin }\end{array}$ & $\begin{array}{l}\text { Immature } \\
\text { fronds }\end{array}$ & eaten fresh or stir-fried & May-Jul & $\begin{array}{l}\text { Whole plant used to treat } \\
\text { rheumatism or for clearing } \\
\text { heat. }\end{array}$ & $* * * *$ \\
\hline $\begin{array}{l}\text { Pyracantha } \\
\text { fortuneana } \\
\text { (Maxim.) H. L. Li }\end{array}$ & Sare & Rosaceae & $\begin{array}{l}\text { Shangri-la, } \\
\text { Weixi and } \\
\text { Deqin }\end{array}$ & Fruits & eaten fresh & Sept-Oct & & $*$ \\
\hline $\begin{array}{l}\text { Pyrus betulifolia } \\
\text { Bunge }\end{array}$ & Reli & Rosaceae & $\begin{array}{l}\text { Shangri-la, } \\
\text { Weixi and } \\
\text { Deqin }\end{array}$ & Fruits & eaten fresh & Aug-Oct & & $*$ \\
\hline $\begin{array}{l}\text { Pyrus calleryana } \\
\text { Decne. }\end{array}$ & Xialie & Rosaceae & $\begin{array}{l}\text { Shangri-la, } \\
\text { Weixi and } \\
\text { Deqin }\end{array}$ & Fruits & eaten fresh & Aug-Oct & & * \\
\hline
\end{tabular}


Table 3 Wild edible plants used by the Tibetans in Shangri-la region, Yunnan Province, China (Continued)

\begin{tabular}{|c|c|c|c|c|c|c|c|c|}
\hline $\begin{array}{c}\text { Pyrus pashia } \\
\text { Buch.-Ham. ex D. } \\
\text { Don }\end{array}$ & Suilun & Rosaceae & $\begin{array}{l}\text { Shangri-la, } \\
\text { Weixi and } \\
\text { Deqin }\end{array}$ & Fruits & eaten fresh & Aug-Oct & & $* *$ \\
\hline $\begin{array}{c}\text { Pyrus } \\
\text { pseudopashia T. } \\
\text { T. Yu }\end{array}$ & Suilun & Rosaceae & $\begin{array}{l}\text { Shangri-la, } \\
\text { Weixi and } \\
\text { Deqin }\end{array}$ & Fruits & eaten fresh & Aug-Sept & & $* *$ \\
\hline $\begin{array}{c}\text { Ramalina } \\
\text { fastigiata (Pers.) } \\
\text { Ach. }\end{array}$ & Shuhua & Ramalinaceae & Whole plant & Whole plant & stir-fried & Jul-Sept & & * \\
\hline $\begin{array}{c}\text { Rheum } \\
\text { likiangense Sam. }\end{array}$ & Mojue & Polygonaceae & $\begin{array}{l}\text { Shangri-la } \\
\text { and Deqin }\end{array}$ & $\begin{array}{l}\text { Young } \\
\text { leaves }\end{array}$ & eaten fresh & Jun-Aug & $\begin{array}{l}\text { Roots used to remove blood } \\
\text { stasis. }\end{array}$ & * \\
\hline $\begin{array}{l}\text { Ribes alpestre } \\
\text { Wall. ex Decne. }\end{array}$ & Suanmiguoguo & Saxifragaceae & $\begin{array}{l}\text { Shangri-la, } \\
\text { Weixi and } \\
\text { Deqin }\end{array}$ & Fruits & eaten fresh and used to prepare local wine & Aug-Sept & $\begin{array}{l}\text { Whole plants used as fence } \\
\text { and hedge plants. }\end{array}$ & $* * *$ \\
\hline $\begin{array}{l}\text { Ribes moupinense } \\
\text { Franch. }\end{array}$ & Hiangshen & Saxifragaceae & $\begin{array}{l}\text { Shangri-la, } \\
\text { Weixi and } \\
\text { Deqin }\end{array}$ & Fruits & eaten fresh and used to prepare local wine & Jul-Oct & $\begin{array}{l}\text { Leaves, stems and fruits used } \\
\text { for weisang. Whole plants used } \\
\text { as fence and hedge plants. }\end{array}$ & $* * *$ \\
\hline $\begin{array}{l}\text { Ribes glaciale } \\
\text { Wall. }\end{array}$ & Niangxu & Saxifragaceae & $\begin{array}{l}\text { Shangri-la, } \\
\text { Weixi and } \\
\text { Deqin }\end{array}$ & Fruits & eaten fresh & Aug-Sept & $\begin{array}{l}\text { Leaves and stems used for } \\
\text { weisang. Whole plants used as } \\
\text { fence and hedge plants. }\end{array}$ & $* * *$ \\
\hline $\begin{array}{l}\text { Rosa omeiensis } \\
\text { Rolfe }\end{array}$ & Xuwabala & Rosaceae & $\begin{array}{l}\text { Shangri-la, } \\
\text { Weixi and } \\
\text { Deqin }\end{array}$ & Fruits & eaten fresh & Jul-Sept & $\begin{array}{l}\text { Whole plants used as fence } \\
\text { and ornamental. }\end{array}$ & *** \\
\hline $\begin{array}{l}\text { Rosa praelucens } \\
\text { Byhouwer }\end{array}$ & Xielermiedu & Rosaceae & Shangri-la & Fruits & eaten fresh & Sept-Oct & $\begin{array}{l}\text { Flowers used for weisang. } \\
\text { Whole plant used as } \\
\text { ornamental. }\end{array}$ & $* * *$ \\
\hline $\begin{array}{l}\text { Rosa soulieana } \\
\text { Crép. }\end{array}$ & Xuwabala & Rosaceae & $\begin{array}{l}\text { Shangri-la, } \\
\text { Weixi and } \\
\text { Deqin }\end{array}$ & Fruits & eaten fresh & Aug-Sept & $\begin{array}{l}\text { Whole plants used as fence } \\
\text { and ornamental. }\end{array}$ & $* *$ \\
\hline $\begin{array}{l}\text { Rubus assamensis } \\
\text { Focke }\end{array}$ & $\begin{array}{l}\text { Hongpai; } \\
\text { Yongde }\end{array}$ & Rosaceae & $\begin{array}{l}\text { Shangri-la, } \\
\text { Weixi and } \\
\text { Deqin }\end{array}$ & Fruits & eaten fresh & Aug-Sept & Whole plants used as fence. & $* *$ \\
\hline $\begin{array}{l}\text { Rubus fockeanus } \\
\text { Kurz }\end{array}$ & $\begin{array}{l}\text { Hongpai; } \\
\text { Yongde }\end{array}$ & Rosaceae & $\begin{array}{l}\text { Shangri-la, } \\
\text { Weixi and } \\
\text { Deqin }\end{array}$ & Fruits & eaten fresh & Aug-Sept & Whole plants used as fence. & * \\
\hline $\begin{array}{l}\text { Rubus niveus } \\
\text { Thunb. }\end{array}$ & $\begin{array}{l}\text { Hongpai; } \\
\text { Yongde }\end{array}$ & Rosaceae & $\begin{array}{l}\text { Shangri-la, } \\
\text { Weixi and } \\
\text { Deqin }\end{array}$ & Fruits & eaten fresh & Aug-Sept & Whole plants used as fence. & $* *$ \\
\hline $\begin{array}{l}\text { Rubus pectinellus } \\
\text { Maxim. }\end{array}$ & Jiaoxumu & Rosaceae & $\begin{array}{l}\text { Shangri-la, } \\
\text { Weixi and } \\
\text { Deqin }\end{array}$ & Fruits & eaten fresh & Aug-Sept & $\begin{array}{l}\text { Leaves and stems used for } \\
\text { weisang. Whole plants used as } \\
\text { fence. }\end{array}$ & $* * *$ \\
\hline $\begin{array}{l}\text { Rubus } \\
\text { pentagonus Wall. } \\
\text { ex Focke }\end{array}$ & $\begin{array}{l}\text { Hongpai; } \\
\text { Yongde }\end{array}$ & Rosaceae & $\begin{array}{l}\text { Shangri-la, } \\
\text { Weixi and } \\
\text { Deqin }\end{array}$ & Fruits & eaten fresh & Aug-Sept & Whole plants used as fence. & $* *$ \\
\hline
\end{tabular}


Table 3 Wild edible plants used by the Tibetans in Shangri-la region, Yunnan Province, China (Continued)

\begin{tabular}{|c|c|c|c|c|c|c|c|c|}
\hline $\begin{array}{l}\text { Rubus } \\
\text { polyodontus } \\
\text { Hand.-Mazz. }\end{array}$ & $\begin{array}{l}\text { Hongpai; } \\
\text { Yongde }\end{array}$ & Rosaceae & $\begin{array}{l}\text { Shangri-la, } \\
\text { Weixi and } \\
\text { Deqin }\end{array}$ & Fruits & eaten fresh & Aug-Sept & Whole plants used as fence. & * \\
\hline $\begin{array}{l}\text { Rubus } \\
\text { rubrisetulosus } \\
\text { Cardot }\end{array}$ & $\begin{array}{l}\text { Hongpai; } \\
\text { Yongde }\end{array}$ & Rosaceae & $\begin{array}{l}\text { Shangri-la, } \\
\text { Weixi and } \\
\text { Deqin }\end{array}$ & Fruits & eaten fresh & Aug-Sept & Whole plants used as fence. & $* *$ \\
\hline $\begin{array}{l}\text { Rubus stans } \\
\text { Focke }\end{array}$ & $\begin{array}{l}\text { Hongpai; } \\
\text { Yongde }\end{array}$ & Rosaceae & $\begin{array}{l}\text { Shangri-la, } \\
\text { Weixi and } \\
\text { Deqin }\end{array}$ & Fruits & eaten fresh & Aug-Sept & Whole plants used as fence. & ** \\
\hline $\begin{array}{l}\text { Sageretia thea } \\
\text { (Osbeck) M. C. } \\
\text { Johnst. }\end{array}$ & Luozi & Rhamnaceae & Deqin & Fruits & eaten fresh & Apr-May & & * \\
\hline $\begin{array}{l}\text { Sambucus } \\
\text { chinensis Lindl. }\end{array}$ & Debangqiongjie & Caprifoliaceae & $\begin{array}{l}\text { Shangri-la, } \\
\text { Weixi and } \\
\text { Deqin }\end{array}$ & Fruits & eaten fresh & Jul-Sept & Aerial parts used as fodder. & *** \\
\hline $\begin{array}{l}\text { Schisandra } \\
\text { rubriflora } \\
\text { (Franch.) Rehder } \\
\text { et E. H. Wilson }\end{array}$ & Wuweizi & Schisandraceae & $\begin{array}{l}\text { Shangri-la, } \\
\text { Weixi and } \\
\text { Deqin }\end{array}$ & Fruits & eaten fresh and used to prepare local wine & Aug-Oct & $\begin{array}{l}\text { Fruits used as antidiarrheic and } \\
\text { for invigorating kidney. Whole } \\
\text { plant used as ornamental. }\end{array}$ & *** \\
\hline $\begin{array}{l}\text { Sinopodophyllum } \\
\text { hexandrum } \\
\text { (Royle) T. S. Ying }\end{array}$ & Agabule & Berberidaceae & $\begin{array}{l}\text { Shangri-la, } \\
\text { Weixi and } \\
\text { Deqin }\end{array}$ & Fruits & eaten fresh & Jul-Sept & $\begin{array}{l}\text { Roots, stems and leaves used } \\
\text { to clear heat. Seeds used to } \\
\text { cure antenatal pain and help } \\
\text { expelling placenta. Whole plant } \\
\text { used as ornamental. }\end{array}$ & $* *$ \\
\hline $\begin{array}{c}\text { Spiranthes } \\
\text { sinensis (Pers.) } \\
\text { Ames }\end{array}$ & Xiaobaiji & Orchidaceae & Shangri-la & Whole plant & stewed with meat and eaten as tonic & Aug-Sept & Whole plants used as fodder. & * \\
\hline $\begin{array}{l}\text { Stachys } \\
\text { kouyangensis } \\
\text { (Vaniot) Dunn } \\
\text { var. franchetiana } \\
\text { (H. Lév.) C. Y. Wu }\end{array}$ & Riganlu & Lamiaceae & $\begin{array}{l}\text { Shangri-la, } \\
\text { Weixi and } \\
\text { Deqin }\end{array}$ & Tubers & boiled or stir-fried & Jun-Sept & Whole plants used as fodder. & * \\
\hline $\begin{array}{l}\text { Taraxacum } \\
\text { mongolicum } \\
\text { Hand.-Mazz. }\end{array}$ & Yongma & Asteraceae & $\begin{array}{l}\text { Shangri-la, } \\
\text { Weixi and } \\
\text { Deqin }\end{array}$ & $\begin{array}{l}\text { Whole } \\
\text { plants }\end{array}$ & boiled or stir-fried & Jun-Aug & Whole plants used as fodder. & *** \\
\hline $\begin{array}{l}\text { Taxillus chinensis } \\
\text { (DC.) Danser }\end{array}$ & Yawakeqi & Loranthaceae & Deqin & Fruits & eaten fresh & Aug-Oct & & ** \\
\hline $\begin{array}{l}\text { Taxillus thibetensis } \\
\text { (Lecomte) Danser }\end{array}$ & Yawakeqi & Loranthaceae & Deqin & Fruits & eaten fresh & Aug-Oct & & *** \\
\hline $\begin{array}{l}\text { Thamnolia } \\
\text { vermicularis Ach. }\end{array}$ & Xiare & Thamnoliaceae & $\begin{array}{l}\text { Shangri-la, } \\
\text { Weixi and } \\
\text { Deqin }\end{array}$ & Whole plant & used for making tea, wine and beverage & Aug-Oct & $\begin{array}{l}\text { Used to tranquilize mind and } \\
\text { clear heat. }\end{array}$ & * \\
\hline Thlaspi arvense L. & Manlancai & Brassicaceae & $\begin{array}{l}\text { Weixi and } \\
\text { Deqin }\end{array}$ & $\begin{array}{l}\text { Young } \\
\text { stems and } \\
\text { leaves }\end{array}$ & stir-fried or used for making pickle & May-Jun & Aerial parts used as fodder. & $* *$ \\
\hline
\end{tabular}


Table 3 Wild edible plants used by the Tibetans in Shangri-la region, Yunnan Province, China (Continued)

\begin{tabular}{|c|c|c|c|c|c|c|c|c|}
\hline Thlaspi arvense L. & Manlancai & Brassicaceae & $\begin{array}{l}\text { Weixi and } \\
\text { Degin }\end{array}$ & Seeds & used for making vegetable oil & Jul-Aug & Aerial parts used as fodder. & ** \\
\hline $\begin{array}{l}\text { Thlaspi } \\
\text { yunnanense } \\
\text { Franch. }\end{array}$ & Manlancai & Brassicaceae & $\begin{array}{l}\text { Shangri-la, } \\
\text { Weixi and } \\
\text { Deqin }\end{array}$ & $\begin{array}{l}\text { Young } \\
\text { stems and } \\
\text { leaves }\end{array}$ & stir-fried or used for making pickle. & May-Jun & Aerial parts used as fodder. & ** \\
\hline $\begin{array}{l}\text { Thlaspi } \\
\text { yunnanense } \\
\text { Franch. }\end{array}$ & Manlancai & Brassicaceae & $\begin{array}{l}\text { Shangri-la, } \\
\text { Weixi and } \\
\text { Deqin }\end{array}$ & Seeds & used for making vegetable oil & Jul-Aug & Aerial parts used as fodder. & ** \\
\hline $\begin{array}{l}\text { Tibetia himalaica } \\
\text { (Baker) H. P. Tsui }\end{array}$ & & Fabaceae & $\begin{array}{l}\text { Deqin, } \\
\text { Shangri-la }\end{array}$ & Roots & eaten fresh & Jun-Aug & Aerial parts used as fodder. & * \\
\hline $\begin{array}{l}\text { Toona sinensis } \\
\text { (Juss.) Roem. }\end{array}$ & & Meliaceae & $\begin{array}{l}\text { Shangri-la, } \\
\text { Weixi and } \\
\text { Deqin }\end{array}$ & Leaf buds & eaten fresh or stir-fried & May-Jun & & ** \\
\hline $\begin{array}{l}\text { Torreya fargesii } \\
\text { Franch. var. } \\
\text { yunnanensis (C. Y. } \\
\text { Cheng et L. K. } \\
\text { Fu) N. Kang }\end{array}$ & Shasongguo & Taxaceae & $\begin{array}{l}\text { Weixi, } \\
\text { Shangri-la }\end{array}$ & seeds & eaten fresh or stir-fried & Sept-Oct & $\begin{array}{l}\text { Leaves and stems used for } \\
\text { weisang. Plants used as fuel- } \\
\text { wood. }\end{array}$ & * \\
\hline $\begin{array}{l}\text { Toxicodendron } \\
\text { succedaneum (L.) } \\
\text { Kuntze }\end{array}$ & Si & Anacardiaceae & $\begin{array}{l}\text { Weixi and } \\
\text { Deqin }\end{array}$ & Fruits & used for making vegetable oil & Jul-Sept & $\begin{array}{l}\text { Wax is extracted from fruits to } \\
\text { use in varnish and polish. }\end{array}$ & * \\
\hline $\begin{array}{l}\text { Toxicodendron } \\
\text { vernicifluum } \\
\text { (Stokes) F. A. } \\
\text { Barkley }\end{array}$ & Si & Anacardiaceae & $\begin{array}{l}\text { Weixi and } \\
\text { Deqin }\end{array}$ & Fruits & used for making vegetable oil & Jul-Sept & $\begin{array}{l}\text { Wax is extracted from fruits for } \\
\text { using in varnish and polish. }\end{array}$ & * \\
\hline $\begin{array}{l}\text { Triosteum } \\
\text { himalayanum } \\
\text { Wall. }\end{array}$ & Sachi & Caprifoliaceae & Shangri-la & Fruits & eaten fresh & Aug-Sept & Aerial parts used as fodder. & * \\
\hline $\begin{array}{l}\text { Typhonium } \\
\text { diversifolium Wall. } \\
\text { ex Schott }\end{array}$ & Banxia & Araceae & Shangri-la & $\begin{array}{l}\text { Young } \\
\text { leaves }\end{array}$ & used for making pickle & Jul-Aug & & ** \\
\hline $\begin{array}{l}\text { Urtica fissa E. } \\
\quad \text { Pritz. }\end{array}$ & Yanglala & Urticaceae & $\begin{array}{l}\text { Shangri-la, } \\
\text { Weixi and } \\
\text { Deqin }\end{array}$ & $\begin{array}{l}\text { Young } \\
\text { stems and } \\
\text { leaves }\end{array}$ & stir-fried & Jun-Jul & & ** \\
\hline $\begin{array}{l}\text { Urtica mairei H. } \\
\text { Lév. }\end{array}$ & Yanglala & Urticaceae & $\begin{array}{l}\text { Shangri-la, } \\
\text { Weixi and } \\
\text { Deqin }\end{array}$ & $\begin{array}{l}\text { Young } \\
\text { stems and } \\
\text { leaves }\end{array}$ & stir-fried & Jun-Jul & & ** \\
\hline $\begin{array}{l}\text { Viburnum } \\
\text { betulifolium } \\
\text { Batalin }\end{array}$ & Ruosi & Caprifoliaceae & $\begin{array}{l}\text { Shangri-la, } \\
\text { Weixi and } \\
\text { Deqin }\end{array}$ & Fruits & eaten fresh and used to prepare local tonic wine & Aug-Sept & & * \\
\hline $\begin{array}{l}\text { Viburnum } \\
\text { kansuense Batalin }\end{array}$ & Ruosi & Caprifoliaceae & $\begin{array}{l}\text { Shangri-la, } \\
\text { Weixi and } \\
\text { Deqin }\end{array}$ & Fruits & eaten fresh and used to prepare local tonic wine & Aug-Sept & & * \\
\hline $\begin{array}{l}\text { Vitis betulifolia } \\
\text { Diels et Gilg }\end{array}$ & Geng & Vitaceae & $\begin{array}{l}\text { Weixi and } \\
\text { Deqin }\end{array}$ & Fruits & eaten fresh & Jul-Oct & Leaves used as fodder. & ** \\
\hline
\end{tabular}


Table 3 Wild edible plants used by the Tibetans in Shangri-la region, Yunnan Province, China (Continued)

\begin{tabular}{|c|c|c|c|c|c|c|c|}
\hline $\begin{array}{l}\text { Zanthoxylum } \\
\text { bungeanum } \\
\text { Maxim. }\end{array}$ & Yemu & Rutaceae & $\begin{array}{l}\text { Shangri-la, } \\
\text { Weixi and } \\
\text { Deqin }\end{array}$ & $\begin{array}{l}\text { Young } \\
\text { stems and } \\
\text { leaves }\end{array}$ & eaten fresh or stir-fried & Apr-May & $* * * *$ \\
\hline $\begin{array}{l}\text { Zanthoxylum } \\
\text { bungeanum } \\
\text { Maxim. }\end{array}$ & Yemu & Rutaceae & $\begin{array}{l}\text { Shangri-la, } \\
\text { Weixi and } \\
\text { Degin }\end{array}$ & Fruits & used as spices & Jul-Sept & $* * * * *$ \\
\hline
\end{tabular}

Frequency: ${ }^{* * * *}>75 \%$ of respondents; ${ }^{* * *}>50 \%$ of respondents; ${ }^{* * *}>1 / 4$ of respondents; ${ }^{* *}>1 / 8$ of respondents; ${ }^{*}<1 / 8$ of respondents, but at least 2 respondents. 
distributed into different life forms, with herbs (43.5\%) and shrubs $(26.8 \%)$ having the most species, similar to a survey conducted in Yunnan Province [17] and another in Hunan Province [21]. The majority of food plants belong to the Rosaceae (34 species), Liliaceae (9), Brassicaceae (9), Araliaceae (6) and Berberidaceae (6). The genera represented by the highest number of species are Rubus (8 species), followed by Maianthemum (6), Berberis (4), Cornus (4), Lindera (4) and Pyrus (4).

The most frequently used parts are fruits, young leaves and stems (Figure 2). This result is similar to other investigations, such as a study of the Shuhi people in the Hengduan Mountains (southwest China) [24], studies in Xishuangbanna, southern Yunnan (China) [26,28] and surveys among Inner Mongolian herdsmen [18]. The preference for wild collected leafy vegetables and fruits over underground plant parts seems to be common among diverse ethnic groups in China and the Himalayan area, and might be due to the ease of collecting above ground parts [24]. Collection period varies from April to August (for young leaves and stems) and July to October (for fruits and seeds). Most plant parts are collected in summer and autumn (Table 3). These plants are often dried in the sun after collection and stored (a very common preserving technique [22]) until winter. Most uses are specific to a particular plant part (such as young leaf, new shoot or ripe fruit), although in a few cases a single plant part has different uses, e.g., seeds of Juglans regia are eaten fresh or used to make vegetable oil. More than one plant part is used for about $7 \%$ of the species. For example, young leaves and stems of Panax japonicus var. major are used as a vegetable, while rhizomes are stewed with meat and eaten as a tonic. Leaves of Thlaspi yunnanense are used as a vegetable, while vegetable oil is made from the seeds.

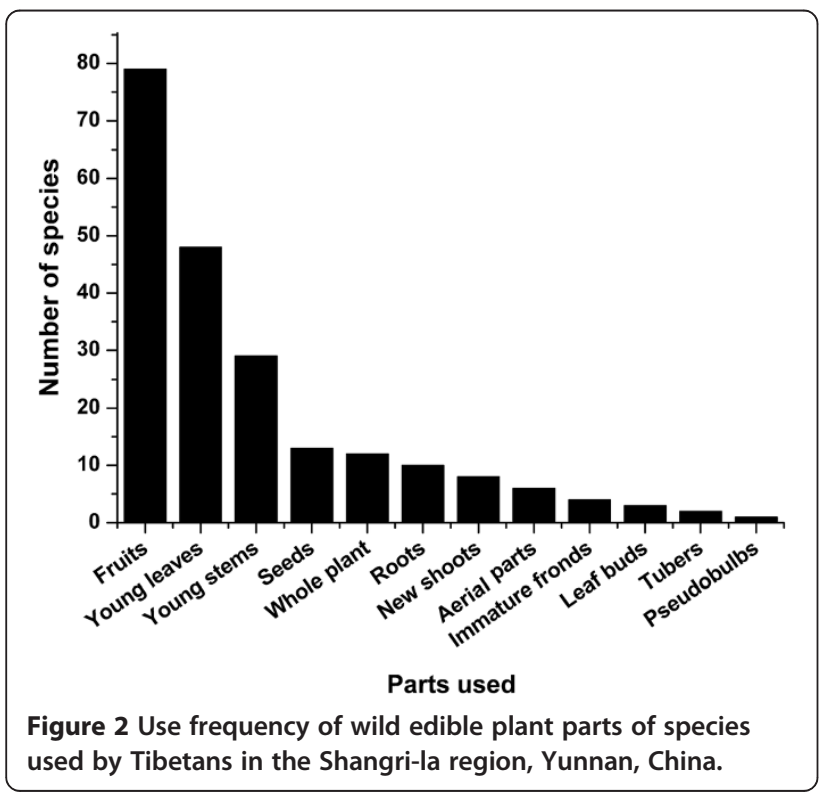

Young leaves, stems and fruits of Berberis amoena, $B$. jamesiana, B. pruinosa and B. eisiensis are eaten fresh. Young stems and leaves of Zanthoxylum bungeanum are boiled or stir-fried, and the fruits as a condiment. Fruits of Berchemia hirtella and B. sinica are eaten fresh and the young leaves to make tea. In total, vegetable (41.9\%) is the most used category followed by fruit (40.8\%) (Table 4). Ripe fruits are often eaten fresh, green leafy vegetative parts (e.g., young leaves and stems) are usually boiled or stir-fried, less commonly they are eaten fresh as salad or added to soups. All these plants are used as ingredients for the hot pot, since Tibetans in this region like hot pot very much.

These wild edible plants play an important role in providing local Tibetans with various vital nutrition elements, such as vitamins and minerals needed to maintain health and promote immunity against disease. For example, butter rice with ginseng fruits is a famous and traditional Tibetan dish. Ginseng fruits are the roots of Potentilla anserina, a perennial herb, and was reported to have low fat, high dietary fiber, all essential amino acids, various mineral elements and vitamins [41]. Other wild vegetables and fruits frequently used by local Tibetans include Maianthemum atropurpureum, Allium ovalifolium, Aralia chinensis, Hippophae rhamnoides subsp. yunnanensis and Amygdalus mira, which are all mentioned by nearly every respondent.

\section{Multiple uses of wild edible plants}

In addition to edible use, $71.4 \%$ of the reported wild edible plants (120 species) have additional uses (Tables 3 and 5). Such species are common in rural areas and are important to local people $[12,42]$. They not only balance the nutritional value of starchy diets (compensating for lack of several vitamins, proteins and minerals), but may also provide pharmacologically active compounds. The multiple uses attest to the importance of these plants for subsistence and as a part of local cultural heritage [12]. Thirtyone species $(18.5 \%)$ are also used as medicine, most are herbs (19 species) or trees (6 species). These medicinal plants are used to treat gastropathy, cough, fever, rheumatism, dysentery, fractures, dyspepsia, hemoptysis, and

Table 4 Specific edible uses of wild edible plants used by Tibetans in Shangri-la region, Yunnan, China

\begin{tabular}{ll}
\hline Specific use & Number of plants \\
\hline vegetable & 80 \\
\hline fruit & 78 \\
\hline wine & 11 \\
\hline vegetable oil & 9 \\
\hline spice & 8 \\
\hline tea & 5 \\
\hline Total & 191 \\
\hline
\end{tabular}


Table 5 Types of multiple uses for edible wild plants utilized by Tibetans in Shangri-la region, Yunnan, China

\begin{tabular}{lll}
\hline Kind of usage & Number of species & Percentage \\
\hline Edible & 168 & 100.0 \\
\hline Fodder & 52 & 31.0 \\
\hline Medicinal & 31 & 18.5 \\
\hline Fence & 22 & 13.1 \\
\hline Ornamental & 11 & 6.5 \\
\hline Weisang & 10 & 6.0 \\
\hline Fuel-wood & 9 & 5.4 \\
\hline Construction & 4 & 2.4 \\
\hline
\end{tabular}

${ }^{\mathrm{a}}$ The religious rite of burning offerings for smoke, which plays an important role in local Tibetan's daily life.

asthma. For a few species, the same part is not only used as food, but is also used for medicinal purposes. For example, the roots of Anemone rivularis are stewed with meat and eaten as tonic by local people, and the decoction of them are used to treat bronchitis.

WEPs can provide resources for future exploitation of new health foods. As living standards improve, there is a globally increased demand for healthy and safe food [21]. Compared to conventional, cultivated vegetables, wild food plants require less care, are not affected by pesticide pollution, and are a rich source of micronutrients.

However destructive harvesting is a significant concern and in the present study this was documented to occur in at least 21 species used for medicine, the underground parts (root, tuber and corm) of fourteen species and the whole plant of seven species. This manner of harvest may have a serious consequence from both the survival of plants and from an ecological point of view [43]. The conservation and sustainable utilization of species with multiple uses should be taken into consideration.

Fifty-two species (31\%) were used as fodder. For example Potentilla coriandrifolia var. dumosa is regarded as high-quality forage at high altitude $(3500-4300 \mathrm{~m})$. Further study of its nutrient composition can be done in order to understand the rationale for its usage and development potential.

Ten species have cultural significance in a religious rite named weisang, during which specific plants are burned for smoke. These are Adenophora khasiana, Aralia chinensis, Cerasus conadenia, Pinus armandii, Pistacia weinmanniifolia, Ribes moupinense, Ribes glaciale, Rosa praelucens, Rubus pectinellus and Torreya fargesii var. yunnanensis. This rite plays an important role in Tibetans' daily life, and it is said that the fragrance in the smoke can not only make the mountain god pleased, but also wash dirty things away from people. Tibetans pray for good harvest, good fortune, happiness and prosperity in this manner.
"Most preferred" species and their commercial potential Besides food value, the recorded species provide the possibility to supplement household income of rural people with limited cash income opportunities [44]. In our survey, the most preferred plants (mentioned by more than $50 \%$ of respondents) include Maianthemum, Allium, Aralia, Arundinaria faberi, Fargesia melanostachys, Pteridium aquilinum var. latiusclum, Matteuccia struthiopteris, Zanthoxylum bungeanum, Ligusticum daucoides, Hippophae rhamnoides subsp. yunnanensis and Pistacia weinmanniifolia. All these plants are collected from remote mountains by local people and traded in local markets, which provides the possibility to increase the income of rural people with low cash income.

Maianthemum species (zhuyecai or "bamboo-leaved vegetable") are the most frequently mentioned wild vegetable. In Diqing Prefecture, the leaves of six species are eaten (M. atropurpureum, $M$. forrestii, $M$. henryi, $M$. oleraceum, $M$. purpureum and $M$. tatsienense). They are added to soups, stir-fried with bacon or eaten raw as salad. Several studies have focused on the nutritional analysis of zhuyecai and found they contained higher amount of protein, essential amino acids, vitamin $C$ and mineral elements compared with some common vegetables [45-47]. Although local people do not use them as medicine, Maianthemum species were reported for medicinal use since ancient times. For instance, M. japonica and $M$. henryi are employed to treat kidney diseases, activate blood circulation and alleviate pain [44,48]. $M$. atropurpurea contains a variety of steroidal saponins and nucleosides which may possess anti-tumor activities [49-51]. Three new steroidal saponins having cytotoxic properties against human cancer cells were isolated from M. japonica [52]. Zhuyecai also has commercial value. In the market the price varied from $12 \mathrm{CNY}$ (Chinese yuan) to $40 \mathrm{CNY}$ (ca. $1 \mathrm{USD}=6.5 \mathrm{CNY}$ ) per kilogram (fresh weight) from April to June, an important source of cash income. And in restaurants, one dish (prepared from about $500 \mathrm{~g}$ ) costs 18-38 CNY during another season.

Another renowned edible plant, shutoucai, includes two species, Aralia caesia and A. chinensis. Leaf buds and young leaves are edible and are collected from April to May. Researchers have reported that the tender shoots of $A$. chinensis contain many oleanolic acids and seven essential amino acids [53]. One local company intends to exploit this wild vegetable commercially. Hippophae rhamnoides subsp. yunnanensis, endemic to the Qinghai-Tibet Plateau, has both food and medicinal values. Its fruits are eaten fresh or used to make beverage and wine, and also used to treat cough and invigorate the circulation of blood.

In the present study, we found that taste is the first criterion for all types of food plants, in agreement with other surveys [54]. However, taste itself is not strong 
enough to construct a reliable priority list for future conservation, domestication and exploitation. Further detailed nutrition analysis and phytochemical investigation should be undertaken to comprehensively evaluate food and medicinal value of these "most preferred" plants, which could provide scientific and important information.

It is generally believed that local people are more likely to support and participate in conservation initiatives if they can receive direct benefits from such efforts [55]. If managed sustainably, these plants could be a good means of income generation for rural communities. Market surveys, value chain analyses and the risk of overexploitation should be assessed thoroughly $[13,56]$. Maianthemum populations (zhuyecai) are becoming rare in Shangri-la County although there were rich resources 20 years ago. Uprooting and harvesting the entire plant during collection were observed and identified as causes of decline for Sinopodophyllum hexandrum, Aristolochia delavayi, Megacarpaea delavayi and Codonopsis pilosula var. handeliana. Because few people in this area are aware of sustainable harvesting, the conservation and proper utilization of these species should be taught.

\section{Crop wild relatives for genetic improvement and crop production}

Crop wild relatives (CWRs) are species that are closely related to crops including crop progenitors. These wild relatives of domesticated crops may provide genes having higher resistance to adverse circumstance that could prove particularly important in response to global climate change, which will undoubtedly alter the environmental conditions under which our crops grow and dramatically impact agriculture $[4,57,58]$. CWRs are also of great importance to maintain the productivity and stability of traditional agroecosystems $[59,60]$. Conservation of these species ensures that diverse genetic resources are preserved and could be used in the improvement of crops as a contribution to $21^{\text {st }}$ century food security $[4,7,8]$. The main options for CWRs conservation are ex situ in gene banks and in situ in the natural or farmed environment $[59,61,62]$. It is widely recognized that in situ is necessary to conserve the full range of genetic diversity inherent in and between plant populations, with ex situ techniques as a backup [58]. Taxon inventory is the starting point for in situ conservation which provides the baseline data critical for biodiversity assessment and monitoring [63]. Some of the wild relatives of fruit, vegetable and spice crops documented in this study are species of Actinidia, Allium, Amaranthus, Amygdalus, Arctium, Armeniaca, Capsella, Cerasus, Crataegus, Dioscorea, Diospyros, Eriobotrya, Foeniculum, Fragaria, Hippophae, Juglans, Malus, Mentha, Pyrus, Toona, Vitis and Zanthoxylum. Take Amygdalus mira as an example. Due to its advantageous traits, such as high adaptability and longevity, resistance to disease and tolerance to drought and cold, it could be a genetic resource for peach improvement. Another case is Pyrus betulifolia, which is usually used as stock to graft various pear cultivars. It is drought resistant, cold tolerant and long living, making it a good candidate for providing useful genes to improve the quality of pears. Young leaves of Allium ovalifolium could be eaten as vegetables, and leaves are relatively larger than those of other Chinese chives. Thus, it might be used as a source for breeding new variety of chives. Two other species, Rosa omeiensis and $R$. praelucens have edible and ornamental uses and exhibit high cold tolerance. They may provide beneficial genes for future study and exploitation in developing new crops.

\section{Issues of conservation}

Wild edible plant species are threatened by various natural causes and human activities [4,34]. Extreme weather caused by global climate change, such as heavy snow and severe droughts, has resulted in the decrease and even loss of many wild food plant populations. Various human activities such as land use change, habitat destruction, overharvesting and over-grazing, are major threats. In recent years, with the construction of roads, airports, reservoirs and other infrastructure, wild habitats for edible plants were severely impacted. Unsustainable harvesting of food plant species with good market price also contributes to a decrease of these plants.

Threats are not only limited to wild food plants themselves, the traditional knowledge associated with WEPs is also endangered. Therefore, systematic documentation of indigenous knowledge and biological resources is of great significance [55,64]. Along with economic development and increasing income, only a few people want to collect wild edible plants. The younger generation is becoming less interested in them, thus causing the loss of traditional knowledge. In Shangri-la County tourism is booming and local people eagerly want to serve as guides or drivers in tourist areas to pursue more money. With the convenience of transportation, residents can buy much more vegetables from the markets than ever before and do not need to collect wild species. However, in more remote rural communities where transportation is still inconvenient and people seldom go to the market, indigenous knowledge about WEPs is relatively intact. In Deqin County much land has been converted to grape cultivation to develop a wine industry and agricultural chemicals are used frequently, causing the decrease of various wild edible species, and even cultivation of the very important species, hull-less barley, Hordeum vulgare, the staple food of Tibetan communities $[65,66]$ is threatened. During our survey we found that most people are reluctant to cultivate hull-less barley now because planting grapes can bring more cash income. 


\section{Conclusion}

This paper is the first ethnobotanical study of wild food plants used by local Tibetans in Diqing Tibetan Autonomous Prefecture. As plant resources in this area are rather plentiful, and under the influence of other ethnic groups, local Tibetans not only cultivate various crops, but also collect wild edible plants as food. Our survey showed the diversity of WEPs and related indigenous knowledge in this area.

Different parts of plants are used by local people, and the most frequently used parts were fruits, young leaves and stems. These plants have different specific food uses, with leafy vegetable uses being most frequent, followed by fruit uses. WEPs provide food and nutrients to local communities, such as essential amino acids, various vitamins and minerals which are needed to keep healthy and enhance immunity against diseases and infections.

If properly harvested, WEPs could be the source of cash income for local people with low cash income because they are enjoyed by local people very much and often traded in markets. Furthermore, with the increased demand for green, healthy and safe food in modern society, wild food resources have attracted global interest because they are pollution-free and contain numerous important micronutrients and pharmacologically active substances. In order to properly utilize the wild food resources, we have some suggestions: 1) properly exploit and improve conservation and management of wild food plants; 2) focus on scientific research on wild food resources; 3) protect the natural environment and habitat for wild food plants.

In addition to food value, more than $50 \%$ of recorded plants have medicinal, ornamental, and cultural and other uses that are important in local Tibetan culture. Furthermore, some are crop wild relatives and could provide useful genes for crop improvement, which may have significant consequence on global food security. However, along with the development of economy, these multi-valued resources are threatened by human activities and natural causes, and associated traditional knowledge is eroding rapidly. Therefore, sustainable management of these resources as well as conserving biodiversity is of the utmost importance.

In a word, our ethnobotanical surveys provide data and information basis for conservation and sustainable utilization of local wild edible plants, and also contribute to preserve cultural and genetic diversity in Diqing Tibetan Autonomous Prefecture.

\section{Competing interests}

The authors declare that they have no competing interests.

\section{Authors' contributions}

CLL designed the study. YJ, BL and JXZ performed the field survey. YJ drafted the manuscript, BL revised the manuscript. CLL revised and finalized the manuscript. All authors read and approved the final manuscript.

\section{Acknowledgements}

We are thankful to the local people for their assistances in the field investigations and for sharing their valuable knowledge. Dr. Kendrick Marr from the Royal British Columbia Museum in Victoria, BC, Canada, kindly helped to edit the English and provided professional comments. We are very grateful to his assistances. Members of Ethnobotanical Laboratory at Minzu University of China, and Research Group of Ethnobotany at Kunming Institute of Botany, Chinese Academy of Sciences, participated in the field work and discussion. This research was funded by the National Science Foundation of China (31161140345), the Ministry of Education of China through its 111 and 985 projects (B08044, MUC98506-01000101 \& MUC985-9), and the Asian COPE Program of Japan Society for the Promotion of Science (JSPS/AP/ 109080).

\section{Author details}

${ }^{1}$ College of Life and Environmental Sciences, Minzu University of China, Beijing 100081, PR China. ${ }^{2}$ School of Agronomy and Biotechnology, Yunnan Agricultural University, Kunming 650201, PR China. ${ }^{3}$ Kunming Institute of Botany, Chinese Academy of Sciences, Kunming 650201, PR China.

Received: 23 January 2013 Accepted: 5 April 2013

Published: 19 April 2013

\section{References}

1. Lulekal E, Asfaw Z, Kelbessa E, Van Damme P: Wild edible plants in Ethiopia: a review on their potential to combat food insecurity. Africa Focus 2011, 24:71-121.

2. Heywood VH: Ethnopharmacology, food production, nutrition and biodiversity conservation: towards a sustainable future for indigenous peoples. J Ethnopharmacol 2011, 137:1-15.

3. Seal T: Evaluation of nutritional potential of wild edible plants, traditionally used by the tribal people of Meghalaya state in India. Amer J Plant Nutr Fertil Tech 2012, 2:19-26.

4. Uprety Y, Poudel R, Shrestha K, Rajbhandary S, Tiwari N, Shrestha U, Asselin $\mathrm{H}$ : Diversity of use and local knowledge of wild edible plant resources in Nepal. J Ethnobiol Ethnomed 2012, 8:16.

5. Ghorbani A, Langenberger G, Sauerborn J: A comparison of the wild food plant use knowledge of ethnic minorities in Naban River Watershed National Nature Reserve, Yunnan, SW China. J Ethnobiol Ethnomed 2012, 8:17.

6. Menendez-Baceta G, Aceituno-Mata L, Tardío J, Reyes-García V, Pardo-de-Santayana M: Wild edible plants traditionally gathered in Gorbeialdea (Biscay, Basque Country). Genet Resour Crop Evol 2012, 59:1329-1347.

7. Ford-Lloyd BV, Schmidt M, Armstrong SJ, Barazani O, Engels J, Ge S, Hadas R, Hammer K, Kell SP, Kang D, Khoshbakht K, Li Y, Long CL, Lu BR, Ma KP, Nguyen VT, Qiu LJ, Wei W, Zhang ZW, Maxted N: Crop wild relativesundervalued, underutilized and under threat? BioScience 2011, 61:559-565.

8. Pandey A, Tomer AK, Bhandari DC, Pareek SK: Towards collection of wild relatives of crop plants in India. Genet Resour Crop Evol 2008, 55:187-202.

9. Misra S, Maikhuri R, Kala C, Rao K, Saxena K: Wild leafy vegetables: A study of their subsistence dietetic support to the inhabitants of Nanda Devi Biosphere Reserve, India. J Ethnobiol Ethnomed 2008, 4:15.

10. Arenas P, Scarpa GF: Edible wild plants of the chorote Indians, Gran Chaco, Argentina. Bot J Linn Soc 2007, 153:73-85.

11. Joshi N, Kehlenbeck K, Maass BL: Traditional, neglected vegetables of Nepal: Their sustainable utilization for meeting human needs, Conference on International Agricultural Research for Development, Tropentag. 2007:1-10.

12. Shrestha PM, Dhillion SS: Diversity and traditional knowledge concerning wild food species in a locally managed forest in Nepal. Agroforest Syst 2006, 66:55-63.

13. Termote C, Van Damme P, Dhed'a Djailo B: Eating from the wild: Turumbu, Mbole and Bali traditional knowledge on non-cultivated edible plants, District Tshopo, DR Congo. Genet Resour Crop Evol 2011, 58:585-618.

14. Keller GB, Mndiga H, Maass BL: Diversity and genetic erosion of traditional vegetables in Tanzania from the farmer's point of view. Plant Genet Resour Charact Util 2005, 3:400-413.

15. Tardío J, Pardi-De-Santayana M, Morales R: Ethnobotanical review of wild edible plants in Spain. Bot J Linn Soc 2006, 152:27-71.

16. Luczaj L, Zovko Končić M, Miličević T, Dolina K, Pandža M: Wild vegetable mixes sold in the markets of Dalmatia (southern Croatia). J Ethnobiol Ethnomed 2013, 9:2. 
17. Ghorbani A, Langenberger G, Liu JX, Wehner S, Sauerborn J: Diversity of medicinal and food plants as non-timber forest products in Naban River Watershed National Nature Reserve (China): implications for livelihood improvement and biodiversity conservation. Econ Bot 2012, 66:178-191.

18. Khasbagan, Huai HY, Pei SJ: Wild plants in the diet of arhorchin mongol herdsmen in Inner Mongolia. Econ Bot 2000, 54:528-536.

19. Wujisguleng $W$, Khasbagen $K$ : An integrated assessment of wild vegetable resources in Inner Mongolian Autonomous Region, China.

J Ethnobiol Ethnomed 2010, 6:34.

20. Huber FK, Ineichen R, Yang YP, Weckerle CS: Livelihood and conservation aspects of non-wood forest product collection in the Shaxi Valley, southwest china. Econ Bot 2010, 64:189-204.

21. Zou XB, Huang FQ, Hao LM, Zhao JW, Mao HP, Zhang JC, Ren SY: The socio-economic importance of wild vegetable resources and their conservation: a case study from China. Kew Bull 2010, 65:577-582.

22. Kang YX, Łuczaj $Ł$, Ye S, Zhang SJ, Kang J: Wild food plants and wild edible fungi of Heihe valley (Qinling Mountains, Shaanxi, central China): herbophilia and indifference to fruits and mushrooms. Acta Soc Bot Pol 2012, 81:405-413.

23. Wang $X Y$, Du XL: Recent status of the development and strategies of exploitation of non-wood forest products in China. Forest Res (China) 1997, 10:199-205.

24. Weckerle CS, Huber FK, Yang YP, Sun WB: Plant knowledge of the Shuhi in the Hengduan Mountains, southwest China. Econ Bot 2006, 60:3-23.

25. Wu X: "Turning waste into things of value": Marketing fern, kudzu, and Osmunda in Enshi Prefecture, China. J Dev Soc 2003, 19:433-457.

26. Xu YK, Tao GD, Liu HM, Yan KL, Dao XS: Wild vegetable resources and market survey in Xishuangbanna, southwest China. Econ Bot 2004, 58:647-667.

27. Zhang XP, Wu ZL, Li Y, Liu F, Wang JQ: Investigation on species resources and utilization of wild vegetable in Nabanhe Watershed Nature Reserve, Xishuangbanna (in Chinese with English abstract). J Southwest Forestry College 2004, 24:21-24

28. Chen J, Su YC, Chen GQ, Wang WD: Ethnobotanical studies on wild edible fruits in Southern Yunnan: folk names; nutritional value and uses. Econ Bot 1999, 53:2-14.

29. Myers N, Mittermeier RA, Mittermeier CG, Da Fonseca GAB, Kent J: Biodiversity hotspots for conservation priorities. Nature 2000, 403:853-858.

30. Liu YC, Dao ZL, Yang CY, Liu YT, Long CL: Medicinal plants used by Tibetans in Shangri-la, Yunnan, China. J Ethnobiol Ethnomed 2009, 5:15.

31. The Compilation Committee of the Records of Diqing Tibentan Autonomous Prefecture: The Records of Diqing Tibetan Autonomous Prefecture. Kunming: The Nationality Press of Yunnan; 2003.

32. Liu ZY: Inheritance and vicissitude of the Tibetan diet-a case of a Tibetan rural community. China Tibetol 2012, 1:92-109. 119.

33. Willson A: Forest conversion and land use change in rural Northwest Yunnan, China. Mountain Res Dev 2006, 26:227-236.

34. Byg A, Salick J: Local perspectives on a global phenomenon-climate change in eastern tibetan villages. Global Environ Change 2009, 19:156-166.

35. Meng R, Zhang LR, Zhang QX: Threatening factors and protection countermeasures to resources of wild ornamental plants in Northwest Yunnan (in Chinese with English abstract). J Hubei Uni 2011, 33:297-303.

36. Khasbagan S: Indigenous knowledge for plant species diversity: a case study of wild plants' folk names used by the Mongolians in Ejina desert area, Inner Mongolia, PR China. J Ethnobiol Ethnomed 2008, 4:2.

37. Alexiades MN, Sheldon JW: Selected guidelines for ethnobotanical research: A field manual. New York: The New York Botanical Garden; 1996.

38. Long CL, Wang JR: The Principle, method and application of participatory rural assessment. Kunming: Yunnan Science and Technology Press; 1996.

39. Chambers R: The origins and practice of participatory rural appraisal. World Dev 1994, 22:953-969.

40. Chambers R: Participatory rural appraisal (PRA): Challenges, potentials and paradigm. World Dev 1994, 22:1437-1454.

41. Sun J, Lu J, Bo H: Analysis and evaluation of dietetic nutrimental of Potentilla anserina Roots. (in Chinese with English abstract). Food Sci 2008, 29:411-414.

42. Etkin NL: Local knowledge of biotic diversity and its conservation in rural Hausaland, Northern Nigeria. Econ Bot 2002, 56:73-88.

43. Teklehaymanot T, Giday M: Ethnobotanical study of wild edible plants of Kara and Kwego semi-pastoralist people in Lower Omo River Valley, Debub Omo Zone, SNNPR, Ethiopia. J Ethnobiol Ethnomed 2010, 6:23.
44. Meng Y, Yang YP, Weckerle CS: Conservation status of Maianthemum species in the Hengduan Mountains: a case study analyzing the impact of new policies on wild collected plant species. Ethnobot Res App/ 2006, 4:167-173.

45. Gui M, Chen B, Shen J, Tian Y: The nutrition value of Smilacina atropurpurea and its exploitation (in Chinese with English abstract). Guihaia 2000, 20:71-74

46. Liu $X$, Zhang J, Fu H: The determination of the trace elements in the leaf and stalk of Smilacina henryi (bekev). Wang ef Tang (in Chinese with English abstract). Studies Trace Elements Health 2003, 20:17-18.

47. Zhang J, Zhou J, Liu X: Nutritional components analysis of the leaf and stalk of Smilacina henryi (bekev). Wang ef Tang from Gaoligongsan Moutains, Yunnan (in Chinese with English abstract). Nat Prod Res Dev 2002, 15:45-47.

48. Wu ZY: Synopsis of Chinese Herbal Medicines (in Chinese). Shanghai: Shanghai Science and Technology Press; 1991

49. Yang SL, Liu XK, Wu H, Wang HB, Qing C: Steroidal saponins and cytoxicity of the wild edible vegetable-Smilacina atropurpurea. Steroids 2009, 74:7-12.

50. Zhang Y, Li HZ, Zhang YJ, Jacob MR, Khan SI, Li XC, Yang CR: Atropurosides A-G, new steroidal saponins from Smilacina atropurpurea. Steroids 2006, 71:712-719.

51. Yang S, Liu X: Nucleosides from Smilacina atropurpurea (in Chinese with English abstract). Chin J Nat Med 2003, 1:196-198.

52. Liu X, Zhang H, Niu XF, Xin W, Qi L: Steroidal saponins from Smilacina japonica. Fitoterapia 2012, 83:812-816.

53. Wang $\mathrm{ZZ}$, Jin $\mathrm{SD}$, Jin $\mathrm{SC}, \mathrm{Hu} \mathrm{JH}$, Zheng $\mathrm{H}$ : Analysis of chemical constituents in medicinal and edible tender shoots of Aralia spp. (in Chinese with English abstract). Acta Nutrimenta Sinica 1999, 21:100-103.

54. Dansi A, Adjatin A, Adoukonou-Sagbadja H, Faladé V, Yedomonhan H, Odou D, Dossou B: Traditional leafy vegetables and their use in the Benin Republic. Genet Resour Crop Evol 2008, 55:1239-1256.

55. Acharya KP, Acharya R: Eating from the wild: indigenous knowledge on wild edible plants in Parroha VDC of Rupandehi district, Central Nepal. Int J Soc Forestry 2010, 3:28-48.

56. Leakey RRB: Potential for novel food products from agroforestry trees: a review. Food Chem 1999, 66:1-14.

57. Hajjar R, Hodgkin T: The use of wild relatives in crop improvement: a survey of developments over the last 20 years. Euphytica 2007, 156:1-13.

58. Maxted N, Kell S: Establishment of a global network for the in situ conservation of crop wild relatives: status and needs. Food and Agriculture Organization of the United Nations: Commission on Genetic Resources for Food and Agriculture; 2009.

59. Meilleur BA, Hodgkin T: In situ conservation of crop wild relatives: status and trends. Biodivers Conserv 2004, 13:663-684.

60. Harlan JR: The possible role of weed races in the evolution of cultivated plants. Euphytica 1965, 14:173-176

61. N'Danikou S, Achigan-Dako EG, Wong JLG: Eliciting local values of wild edible plants in southern Bénin to identify priority species for conservation. Econ Bot 2011, 65:381-395.

62. Khoury C, Laliberté B, Guarino L: Trends in ex situ conservation of plant genetic resources: a review of global crop and regional conservation strategies. Genet Resour Crop Evol 2010, 57:625-639.

63. Heywood V, Casas A, Ford-Lloyd B, Kell S, Maxted N: Conservation and sustainable use of crop wild relatives. Agri Ecosys Environ 2007, 121:245-255

64. Acharya R, Acharya KP: Ethnobotanical study of medicinal plants used by Tharu community of Parroha VDC, Rupandehi district, Nepal. Scientific World 2009, 7:80-84

65. Li YL, Long CL, Kato K, Yang CY, Sato K: Indigenous knowledge and traditional conservation of hulless barley (Hordeum vulgare) germplasm resources in the Tibetan communities of Shangri-la, Yunnan, SW China. Genet Resour Crop Evol 2011, 58:645-655.

66. Guo Y, Li YL, Huang Y, Jarvis D, Sato K, Kato K, Tsuyuzaki H, Chen LJ, Long $\mathrm{CL}$ : Genetic diversity analysis of hulless barley from Shangri-la region revealed by SSR and AFLP markers. Genet Resour Crop Evol 2012, 59(7):1543-1552.

doi:10.1186/1746-4269-9-28

Cite this article as: Ju et al:: Eating from the wild: diversity of wild edible plants used by Tibetans in Shangri-la region, Yunnan, China. Journal of Ethnobiology and Ethnomedicine 2013 9:28. 